\title{
Solid Precipitation Measurement Intercomparison in Bismarck, North Dakota, from 1988 through 1997
}

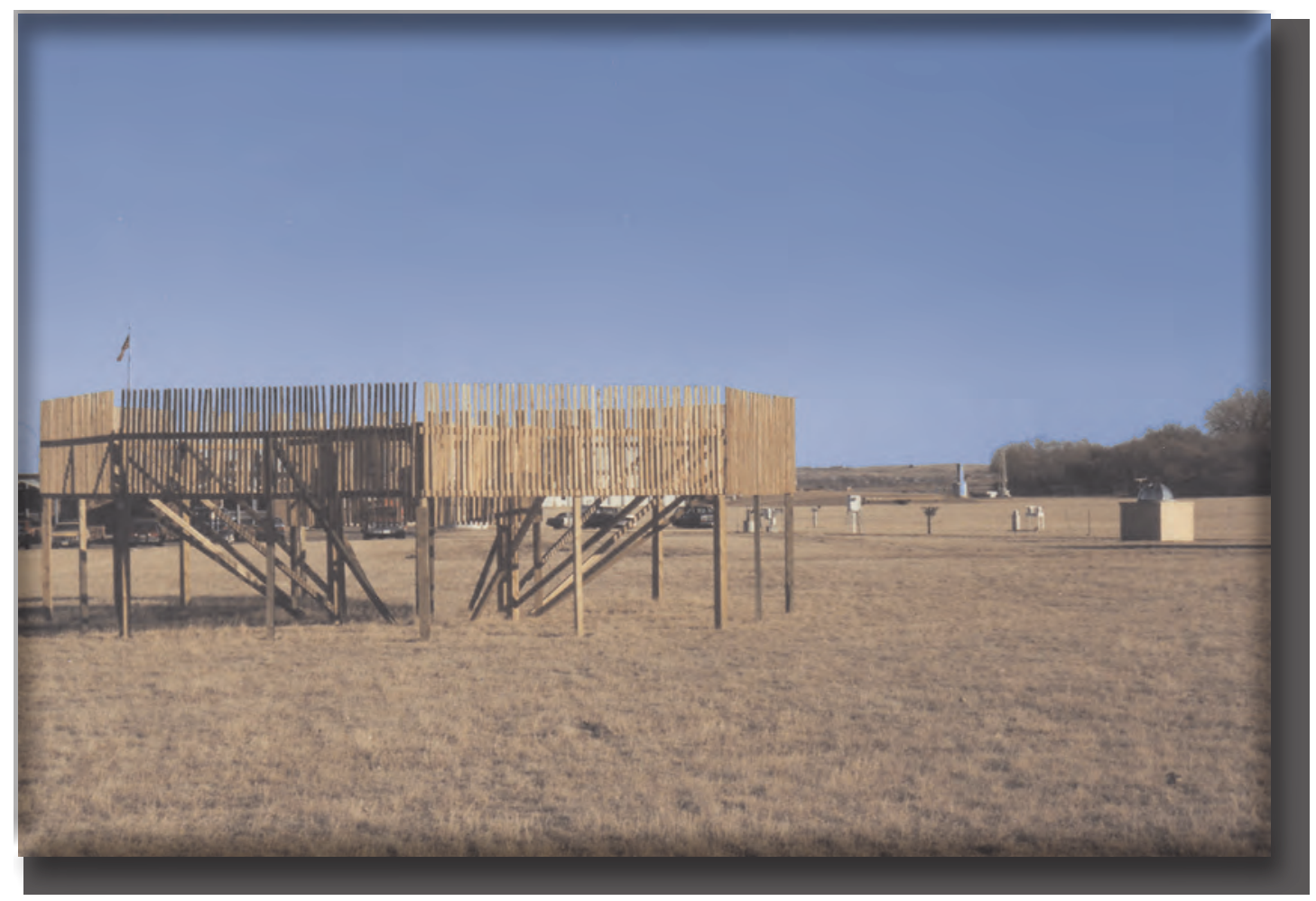

Scientific Investigations Report 2009-5180 
Cover. Double fence intercomparison reference having a Tretyakov gage. 


\section{Solid Precipitation Measurement Intercomparison in Bismarck, North Dakota, from 1988 through 1997}

By Karen R. Ryberg, Douglas G. Emerson, and Kathleen M. Macek-Rowland

Scientific Investigations Report 2009-5180 


\title{
U.S. Department of the Interior \\ KEN SALAZAR, Secretary
}

\author{
U.S. Geological Survey \\ Suzette M. Kimball, Acting Director
}

U.S. Geological Survey, Reston, Virginia: 2009

For more information on the USGS - the Federal source for science about the Earth, its natural and living resources, natural hazards, and the environment, visit http://www.usgs.gov or call 1-888-ASK-USGS

For an overview of USGS information products, including maps, imagery, and publications, visit http://www.usgs.gov/pubprod

To order this and other USGS information products, visit http://store.usgs.gov

Any use of trade, product, or firm names is for descriptive purposes only and does not imply endorsement by the U.S. Government.

Although this report is in the public domain, permission must be secured from the individual copyright owners to reproduce any copyrighted materials contained within this report.

Suggested citation:

Ryberg, K.R., Emerson, D.G., and Macek-Rowland, K.M., 2009, Solid precipitation measurement intercomparison in Bismarck, North Dakota, from 1988 through 1997: U.S. Geological Survey Scientific Investigations Report 2009-5180, $24 \mathrm{p}$. 


\section{Acknowledgments}

The authors acknowledge and thank the National Weather Service, Bismarck, N. Dak., Weather Forecast office for their 10 years of support for this project. 


\section{Contents}

Acknowledgments .......................................................................................................................

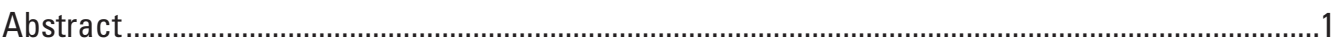

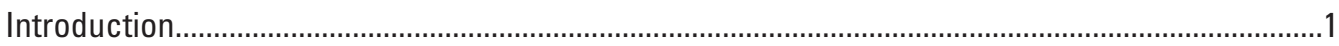

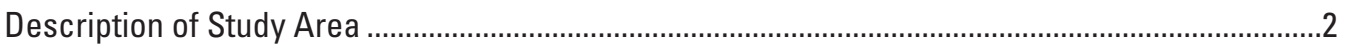

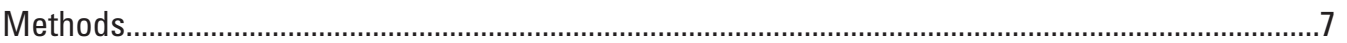

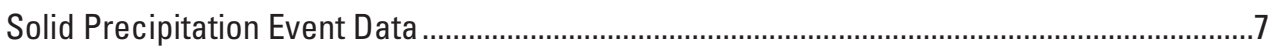

Correction of Data from the Double Fence Intercomparison Reference .................................13

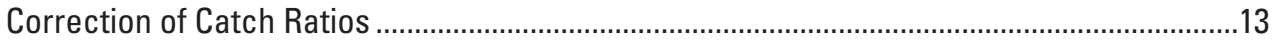

Solid Precipitation Measurement Intercomparison ....................................................................19

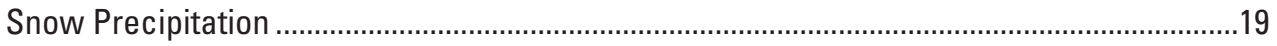

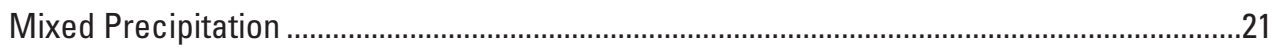

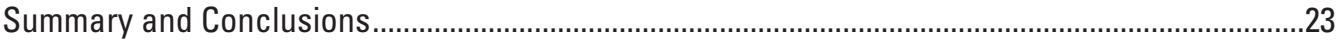

References

\section{Figures}

1-9. Photographs showing:

1. Double fence intercomparison reference having a Tretyakov gage ..........................3

2. Tretyakov precipitation gage ..........................................................................

3. National (National Weather Service) gage equipped with the national standard windshield .....................................................................................................

4. National (National Weather Service) gage without windshield ................................

5. Temperature and humidity sensing system of the National Weather Service.

6. Wind speed and wind direction sensors at 3.0-meter height and wind speed sensor at 1.4-meter height

7. AeroChem Metrics (model 301) automatic sensing wet/dry precipitation

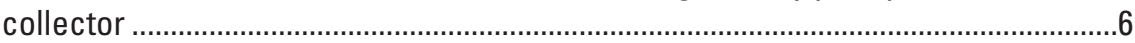

8. National (National Weather Service) gage with double fence shield .......................6

9. National (National Weather Service) gage with a Wyoming windshield ..................7

10-15. Graphs showing:

10. Ratios of gage catch to the corrected double fence intercomparison reference and wind speeds for events in Bismarck, N. Dak., from 1988 through 1997

11. Ratios of gage catch to the corrected double fence intercomparison reference and mean, maximum, and minimum temperatures for events in Bismarck, N. Dak., from 1988 through 1997.

12. Ratios of gage catch to the corrected double fence intercomparison reference and upper air (700-millibar pressure) temperatures in Bismarck, N. Dak., from 1988 through 1997

13. Ratios of gage catch to the corrected double fence intercomparison reference and upper air (700-millibar pressure) wind speed in Bismarck, N. Dak., from 1988 through 1997 
14. Predicted snow catch based on World Meteorological Organization (WMO) equations and equations developed in this study (USGS), and corrected reference gage [double fence intercomparison reference (DFIR)] snow catch for three gages

15. Predicted mixed precipitation catch based on World Meteorological Organization (WMO) equations and corrected reference gage [double fence intercomparison reference (DFIR)] mixed precipitation catch for three gages

\section{Tables}

1. Data collected during solid precipitation measurement intercomparison in Bismarck, N. Dak., from 1988 through 1997.

2. Results of regression analysis of solid precipitation measurement intercomparison data from Bismarck, N. Dak., 1998 through 1997.

3. Solid precipitation measurement intercomparison data from Bismarck, N. Dak., 1988 through 1997, adjusted by regression analysis and World Metrological Organization solid precipitation measurement intercomparison equations...

4. Number of mixed precipitation observations at each gage at Bismarck, N. Dak., from 1988 through 1997

5. Catch ratio equations for mixed precipitation from the World Meteorological Organization solid precipitation measurement intercomparison.. 


\section{Conversion Factors, Datums, and Abbreviations}

\begin{tabular}{lcl}
\hline Multiply & By & To obtain \\
\hline centimeter $(\mathrm{cm})$ & Length & \\
millimeter $(\mathrm{mm})$ & 0.3937 & inch (in.) \\
meter $(\mathrm{m})$ & 0.03937 & inch (in.) \\
kilometer $(\mathrm{km})$ & 3.281 & foot (ft) \\
\hline & 0.6214 & mile (mi) \\
\hline square centimeter $\left(\mathrm{cm}^{2}\right)$ & Area & \\
square centimeter $\left(\mathrm{cm}^{2}\right)$ & 0.1550 & square inch $\left(\mathrm{ft}^{2}\right)$ \\
square meter $\left(\mathrm{m}^{2}\right)$ & 0.001076 & square foot $\left(\mathrm{ft}^{2}\right)$ \\
square meter $\left(\mathrm{m}^{2}\right)$ & $1,550.0031$ & square inches $\left(\mathrm{in}^{2}\right)$ \\
& 10.76 & square foot $\left(\mathrm{ft}^{2}\right)$ \\
\hline pascal $(\operatorname{Pa})$ & Pressure & \\
\hline
\end{tabular}

Temperature in degrees Celsius $\left({ }^{\circ} \mathrm{C}\right)$ may be converted to degrees Fahrenheit $\left({ }^{\circ} \mathrm{F}\right)$ as follows:

$$
{ }^{\circ} \mathrm{F}=\left(1.8 x^{\circ} \mathrm{C}\right)+32
$$

Vertical coordinate information is referenced to the National Geodetic Vertical Datum of 1929 (NGVD 29).

Horizontal coordinate information is referenced to the 1927 North American datum.

Elevation, as used in this report, refers to distance above the vertical datum.

\section{Abbreviations}

CIM0-IX Ninth Session of the Commission for Instruments and Methods of Observation

CR catch ratio

DFIR double fence intercomparison reference

IGRA Integrated Global Radiosonde Archive

NWS National Weather Service

RPD relative percentage difference

USGS U.S. Geological Survey

WMO World Meteorological Organization 


\title{
Solid Precipitation Measurement Intercomparison in Bismarck, North Dakota, from 1988 through 1997
}

\author{
By Karen R. Ryberg, Douglas G. Emerson, and Kathleen M. Macek-Rowland
}

\section{Abstract}

A solid precipitation measurement intercomparison was recommended by the World Meteorological Organization (WMO) and was initiated after approval by the ninth session of the Commission for Instruments and Methods of Observation. The goal of the intercomparison was to assess national methods of measuring solid precipitation against methods whose accuracy and reliability were known. A field study was started in Bismarck, N. Dak., during the 1988-89 winter as part of the intercomparison. The last official field season of the WMO intercomparison was 1992-93; however, the Bismarck site continued to operate through the winter of 1996-97.

Precipitation events at Bismarck were categorized as snow, mixed, or rain on the basis of descriptive notes recorded as part of the solid precipitation intercomparison. The rain events were not further analyzed in this study. Catch ratios (CRs) - the ratio of the precipitation catch at each gage to the true precipitation measurement (the corrected double fence intercomparison reference) — were calculated. Then, regression analysis was used to develop equations that model the snow and mixed precipitation CRs at each gage as functions of wind speed and temperature. Wind speed at the gages, functions of temperature, and upper air conditions (wind speed and air temperature at 700 millibars pressure) were used as possible explanatory variables in the multiple regression analysis done for this study. The CRs were modeled by using multiple regression analysis for the Tretyakov gage, national shielded gage, national unshielded gage, AeroChem gage, national gage with double fence, and national gage with Wyoming windshield.

As in earlier studies by the WMO, wind speed and air temperature were found to influence the CR of the Tretyakov gage. However, in this study, the temperature variable represented the average upper air temperature over the duration of the event. The WMO did not use upper air conditions in its analysis.

The national shielded and unshielded gages where found to be influenced by functions of wind speed only, as in other studies, but the upper air wind speed was used as an explanatory variable in this study. The AeroChem gage was not used in the WMO intercomparison study for 1987-93. The AeroChem gage had a highly varied CR at Bismarck, and a number of variables related to wind speed and temperature were used in the model for the CR. Despite extensive efforts to find a model for the national gage with double fence, no statistically significant regression model was found at the 0.05 level of statistical significance. The national gage with Wyoming windshield had a CR modeled by temperature and wind speed variables, and the regression relation had the highest coefficient of determination $\left(\mathrm{R}^{2}=0.572\right)$ and adjusted coefficient of multiple determination $\left(\mathrm{R}_{a}^{2}=0.476\right)$ of all of the models identified for any gage.

Three of the gage CRs evaluated could be compared with those in the WMO intercomparison study for 1987-93. The WMO intercomparison had the advantage of a much larger dataset than this study. However, the data in this study represented a longer time period. Snow precipitation catch is highly varied depending on the equipment used and the weather conditions. Much of the variation is not accounted for in the WMO equations or in the equations developed in this study, particularly for unshielded gages.

Extensive attempts at regression analysis were made with the mixed precipitation data, but it was concluded that the sample sizes were not large enough to model the CRs. However, the data could be used to test the WMO intercomparison equations. The mixed precipitation equations for the Tretyakov and national shielded gages are similar to those for snow in that they are more likely to underestimate precipitation when observed amounts were small and overestimate precipitation when observed amounts were relatively large. Mixed precipitation is underestimated by the WMO adjustment and the national unshielded gage. Results show that the precision of snow and mixed precipitation measurement varies greatly depending on the equipment used and the weather conditions. Mixed precipitation catch is highly varied, and both mixed and snow catch is highly varied for unshielded gages.

\section{Introduction}

In 1985, the International Workshop on Correction of Precipitation Measurements recommended that the World Meteorological Organization (WMO) organize a solid precipitation measurement intercomparison to assess national 
methods of measuring solid precipitation against methods whose accuracy and reliability were known, including past and current procedures, automated systems, and new methods of observation. The intercomparison was initiated after approval by the ninth session of the Commission for Instruments and Methods of Observation (CIMO-IX). The intercomparison's goal was to

1. determine wind related errors in national methods of measuring solid precipitation,

2. derive standard methods for adjusting solid precipitation measurements, and

3. introduce a reference method of solid precipitation measurement for general use to calibrate any type of precipitation gage (Goodison and others, 1998).

Intercomparison field studies were started in some countries during the 1986-87 winter. The field study in Bismarck, N. Dak., began during the 1988-89 winter. The last official field season of the WMO intercomparison was 1992-93, and data from 1987 through 1993 were analyzed (Goodison and others, 1998). Some sites, including the Bismarck site, continued to operate after 1993. The last field season for the Bismarck site was 1996-97, and data collected at the site after 1992-93 have not been published or analyzed previously.

The WMO intercomparison found that discrepancies of as much as 110 percent existed among snowfall records from various national gages (Yang and others, 2001). On the basis of the intercomparison results, regression equations were developed by the WMO to model the catch ratio (CR) of the various solid precipitation instrumentation. The $\mathrm{CR}$ is a measure of the relative catch efficiency of a gage as compared to a reference gage, defined to be the true measure of precipitation (Goodison and others, 1998). The reference gage for the WMO intercomparison and this study was the double fence intercomparison reference (DFIR) having a Tretyakov gage.

WMO regression equations, based on a combined international dataset collected by the WMO Solid Precipitation Measurement Intercomparison project, were developed for the four most widely used nonrecording gages for measurement of both solid (snow) precipitation and mixed (snow and rain) precipitation. These equations are necessary to obtain comparable measurements of precipitation because of the differing types of national standard precipitation gages operated around the world. The four gages were the Russian Tretyakov gage (shielded), the Hellmann gage (unshielded and shielded), the Canadian Nipher gage (a shielded, nonrecording gage), and the National Weather Service (United States; NWS) 8-inch ( 0.203 meter) standard gage (unshielded and shielded). The precipitation type and gage type combinations resulted in 10 regression equations for $\mathrm{CR}$ at the gages.

The study conducted in Bismarck did not use the Hellmann or Canadian Nipher gages; however, it included additional gages: an AeroChem Metrics gage, an NWS (national) gage with a double fence, and a national gage with a Wyoming windshield. With the additional data available at Bismarck from the extended period of operation, regression equations were developed to model the CR of each of the gages for snow and mixed precipitation. The equations were compared with the WMO published regression equations for the respective gage types (Goodison and others, 1998).

This report describes the solid precipitation measurement intercomparison in Bismarck for data collected from 1988 through 1997. The report (1) publishes the additional Bismarck data that were not part of the WMO intercomparison, (2) describes regression analysis on the Bismarck data that uses the explanatory variables in the WMO intercomparison and additional variables suggested by other research, (3) describes a comparison of the equations developed in this report to the WMO equations, and (4) examines the effectiveness of the regression equations from the WMO study and those in this report for adjusting the Bismarck data. Regression equations also were developed for gages not part of the WMO intercomparison.

\section{Description of Study Area}

Instrumentation was located at an elevation of 502 meters (m) [1,647 feet (ft)] above the National Geodetic Vertical Datum of 1929 (NGVD 29) datum in an open site at the NWS Forecast Office at the Bismarck Municipal Airport, lat $46^{\circ} 46^{\prime} 19^{\prime \prime} \mathrm{N}$., long $100^{\circ} 45^{\prime} 39^{\prime \prime} \mathrm{W}$. The station was on the east bank of the Missouri River in a shallow basin 11.26 kilometers $(\mathrm{km})$ [7 miles (mi)] wide and $17.70 \mathrm{~km}(11 \mathrm{mi})$ long. The site was almost entirely surrounded by low-lying hills.

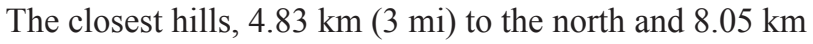
$(5 \mathrm{mi})$ to the southeast, are about 60.96 to $91.44 \mathrm{~m}$ (200 to $300 \mathrm{ft}$ ) high. West, across the Missouri River, the land is more hilly and 91.44 to $182.88 \mathrm{~m}$ (300 to $600 \mathrm{ft}$ ) higher. The topographic features do not have a major effect on climate or prevailing winds (D.G. Emerson, U.S. Geological Survey, written commun., 1990). Instrumentation included:

1. One DFIR having a Tretyakov gage (fig. 1). The gage's orifice was 0.16 meter $(\mathrm{m})$ [6.3 inches (in.)] in diameter, 0.02 square meter $\left(\mathrm{m}^{2}\right)$ [36 square inches $\left(\right.$ in. $\left.\left.^{2}\right)\right]$ in receiving area, and $3.0 \mathrm{~m}(9.8 \mathrm{ft})$ above ground.

2. One Tretyakov precipitation gage (fig. 2). The gage's orifice was $0.16 \mathrm{~m}$ (6.3 in.) in diameter, $0.02 \mathrm{~m}^{2}\left(31 \mathrm{in}^{2}\right)$ in receiving area, and $1.4 \mathrm{~m}(4.6 \mathrm{ft})$ above ground.

3. One national gage (NWS) equipped with the national standard windshield (fig. 3). The national gage was a Belfort Universal Recording Rain Gage (model 5-780). The gage's orifice was $0.203 \mathrm{~m}$ (8.00 in.) in diameter, $0.13 \mathrm{~m}^{2}\left(50 \mathrm{in.}^{2}\right)$ in receiving area, and $1.4 \mathrm{~m}(4.6 \mathrm{ft})$ above ground. The national standard windshield was an Alter-Type Windshield. 


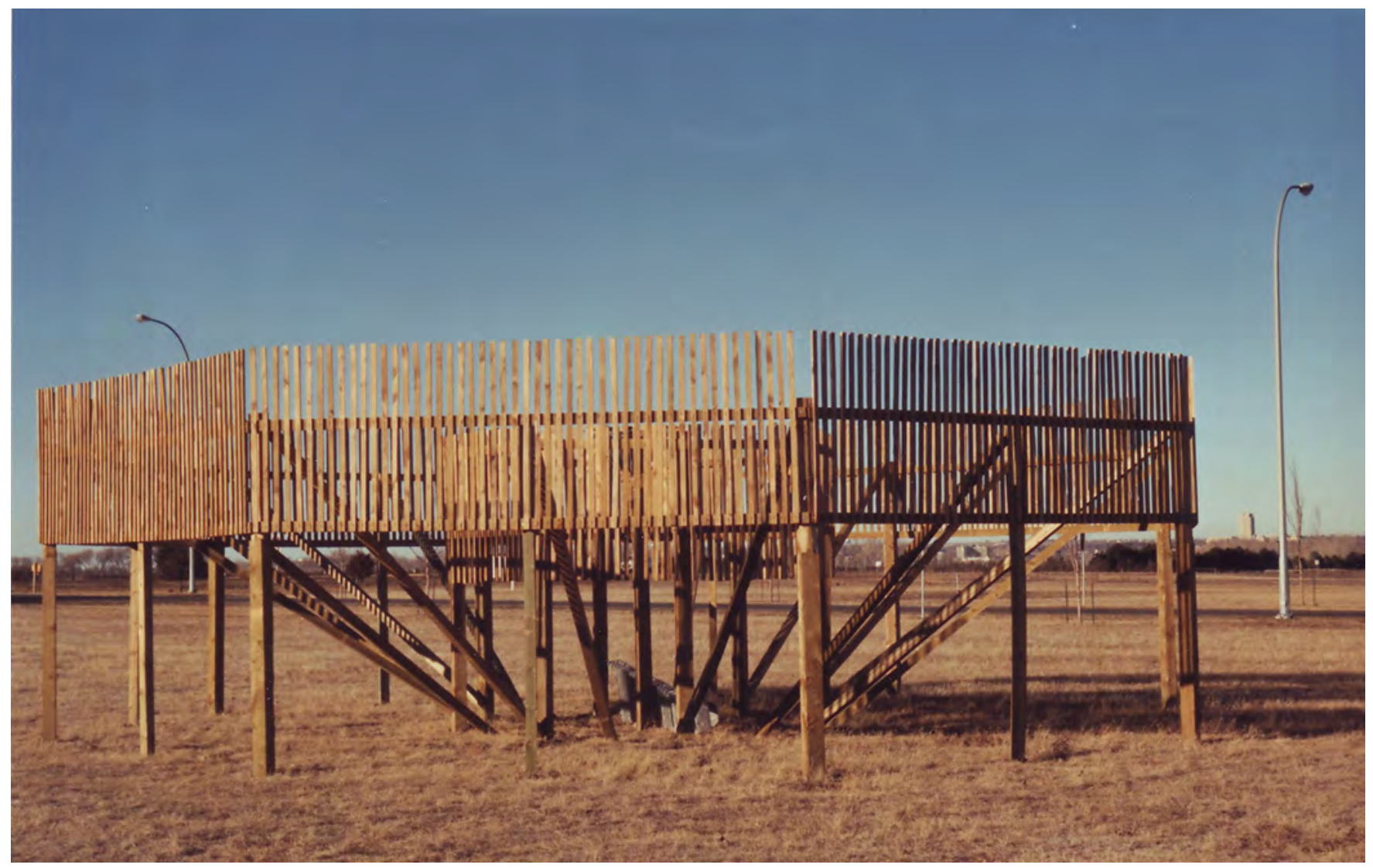

Figure 1. Double fence intercomparison reference having a Tretyakov gage.

4. One national (NWS) gage without windshield (fig. 4). The gage was a Belfort Universal Transmitting Precipitation Gage (model 5915) equipped with an Omnidata Datapod recorder (model DP111). The gage's orifice was $0.203 \mathrm{~m}$ (8.00 in.) in diameter, $0.13 \mathrm{~m}^{2}\left(50 \mathrm{in.}^{2}\right)$ in receiving area,

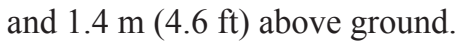

5. The temperature and humidity sensing system of the NWS (fig. 5).

6. Wind speed and wind direction sensors (fig. 6). The wind sensors of the NWS were installed at a height of $6.1 \mathrm{~m}$ $(20 \mathrm{ft})$ above ground and were F-420C systems, Electric Speed Indicator Co., Cleveland, Ohio. A Met-One Wind Direction Sensor (model 024A) and Met-One Wind Speed Sensor (model 014A) were installed at $3.0 \mathrm{~m}(9.8 \mathrm{ft})$ above ground (fig. $6 A$ ), and a Met-One Wind Speed Sensor (model 014A) was installed at $1.4 \mathrm{~m}(4.6 \mathrm{ft})$ above ground (fig. 6B).
7. One AeroChem Metrics (model 301) automatic sensing wet/dry precipitation collector (fig. 7). The orifice was $0.293 \mathrm{~m}$ (11.5 in.) in diameter, $0.067 \mathrm{~m}^{2}\left(104 \mathrm{in.}^{2}\right)$ in receiving area, and $1.4 \mathrm{~m}(4.6 \mathrm{ft})$ above ground.

8. One national (NWS) gage with double fence shield (fig. 8). The gage was a Belfort Universal Transmitting Precipitation Gage (model 5915) equipped with an Omnidata Datapod recorder (model DP111). The gage's orifice was $0.203 \mathrm{~m}$ (8.00 in.) in diameter, $0.13 \mathrm{~m}^{2}\left(50 \mathrm{in}^{2}{ }^{2}\right)$ in receiving area, and $3.0 \mathrm{~m}(9.8 \mathrm{ft})$ above ground.

9. One national (NWS) gage with a Wyoming windshield (fig. 9). The gage was a Belfort Universal Transmitting Precipitation Gage (model 5915) equipped with an Omnidata Datapod recorder (model DP111). The gage's orifice was $0.203 \mathrm{~m}$ (8.00 in.) in diameter, $0.13 \mathrm{~m}^{2}\left(50 \mathrm{in}^{2}{ }^{2}\right)$ in receiving area, and $1.4 \mathrm{~m}(4.6 \mathrm{ft})$ above ground. 


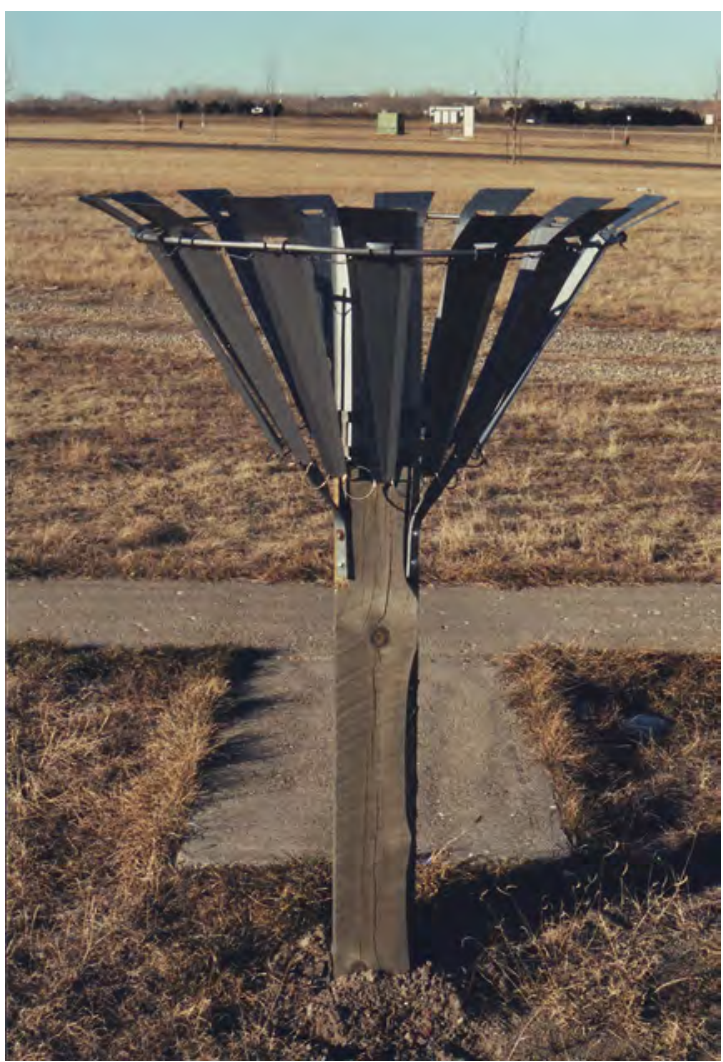

Figure 2. Tretyakov precipitation gage.

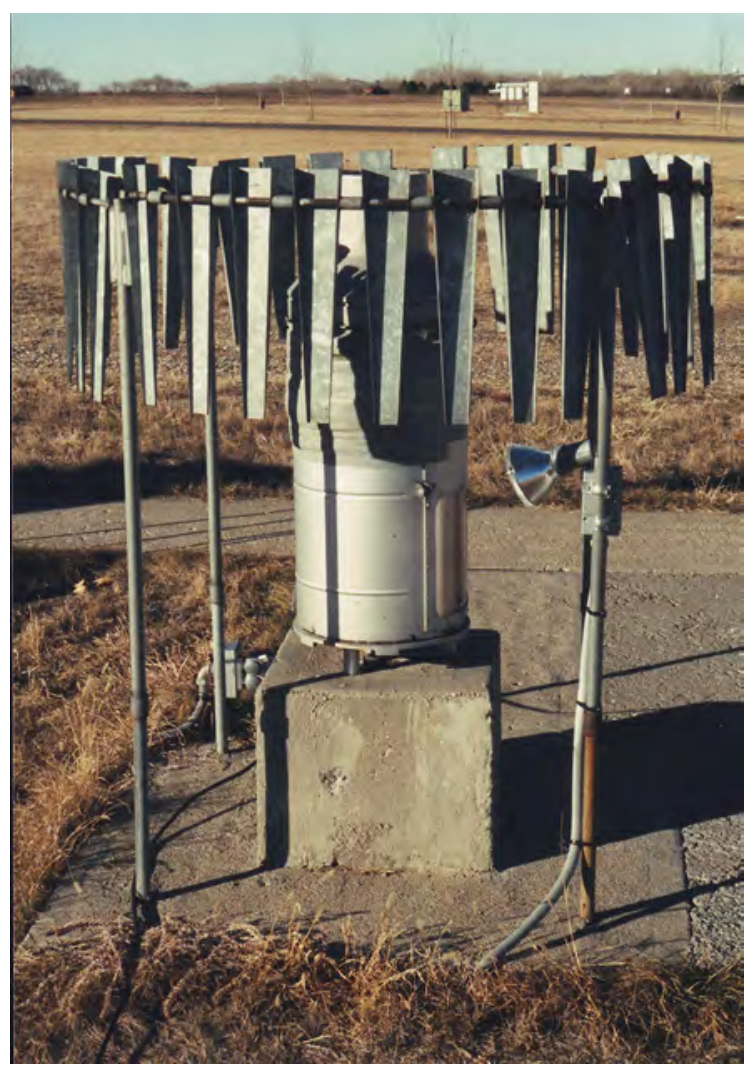

Figure 3. National (National Weather Service) gage equipped with the national standard windshield.

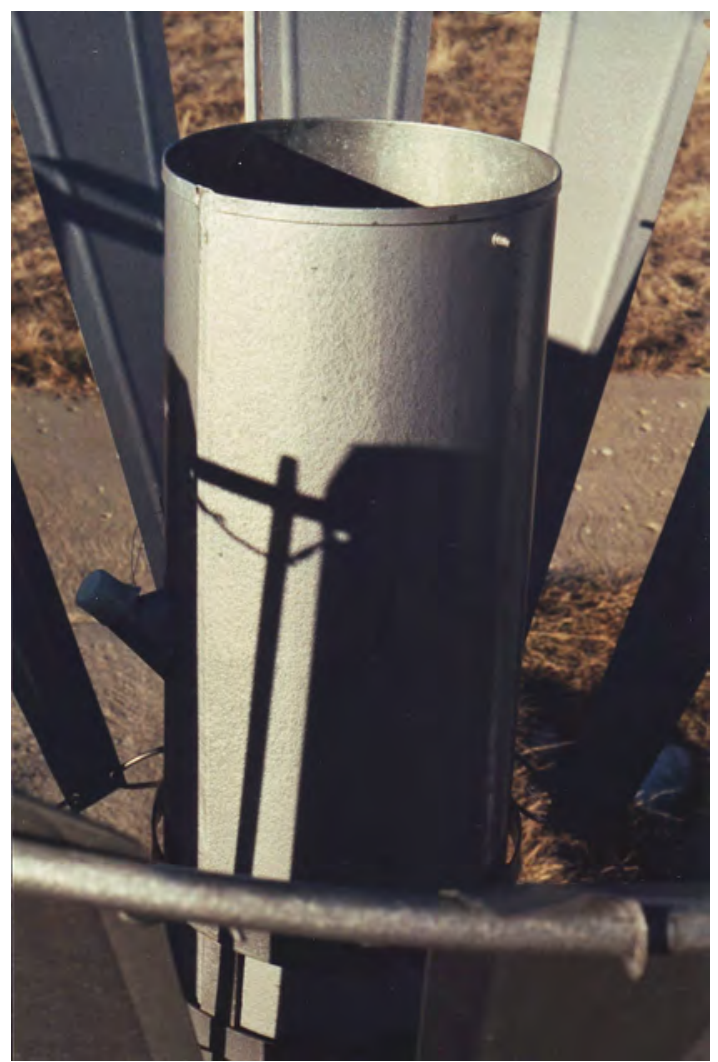

Figure 2. Continued.

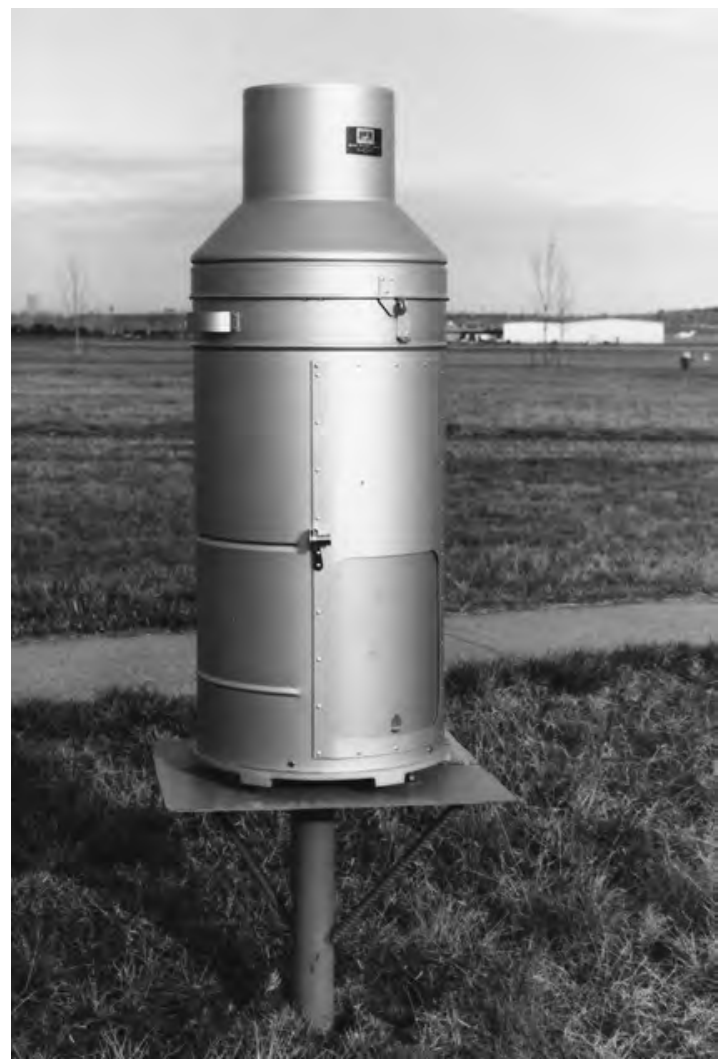

Figure 4. National (National Weather Service) gage without windshield. 


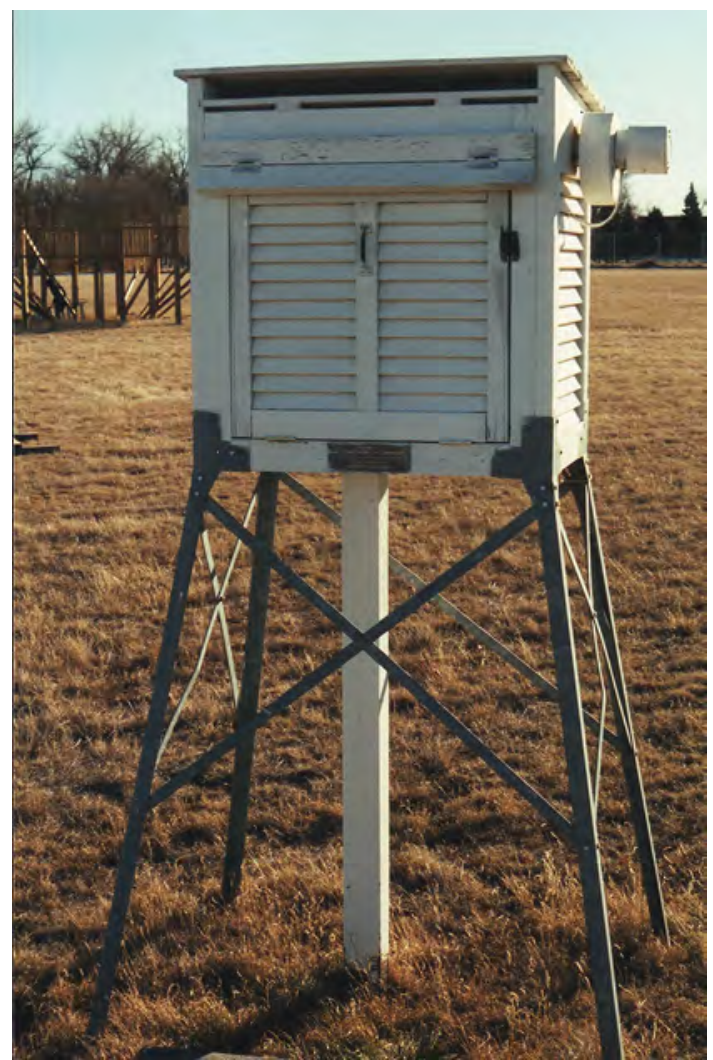

Figure 5. Temperature and humidity sensing system of the National Weather Service.
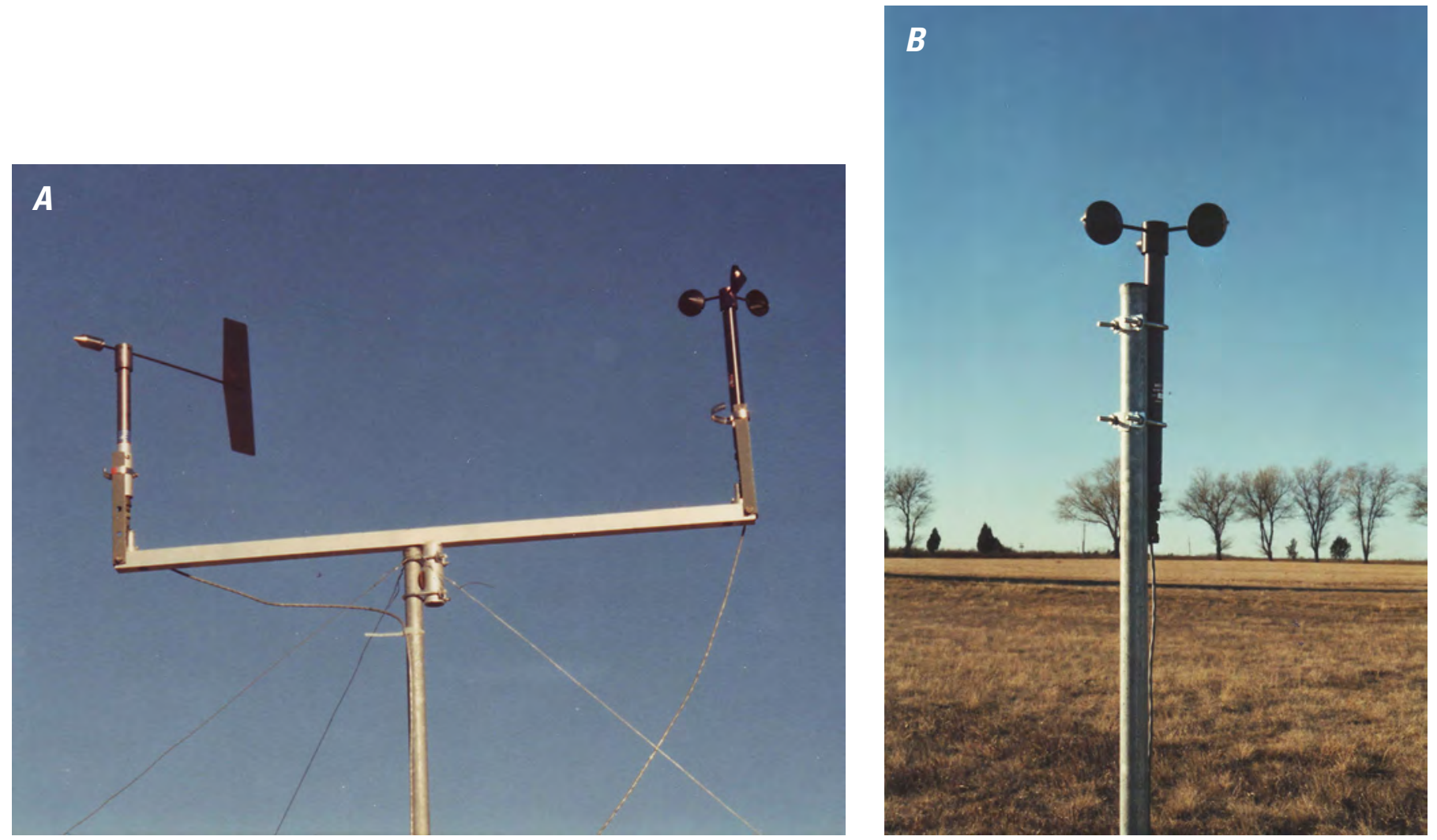

Figure 6. Wind speed and wind direction sensors at 3.0-meter height $(A)$ and wind speed sensor at 1.4-meter height $(B)$. 


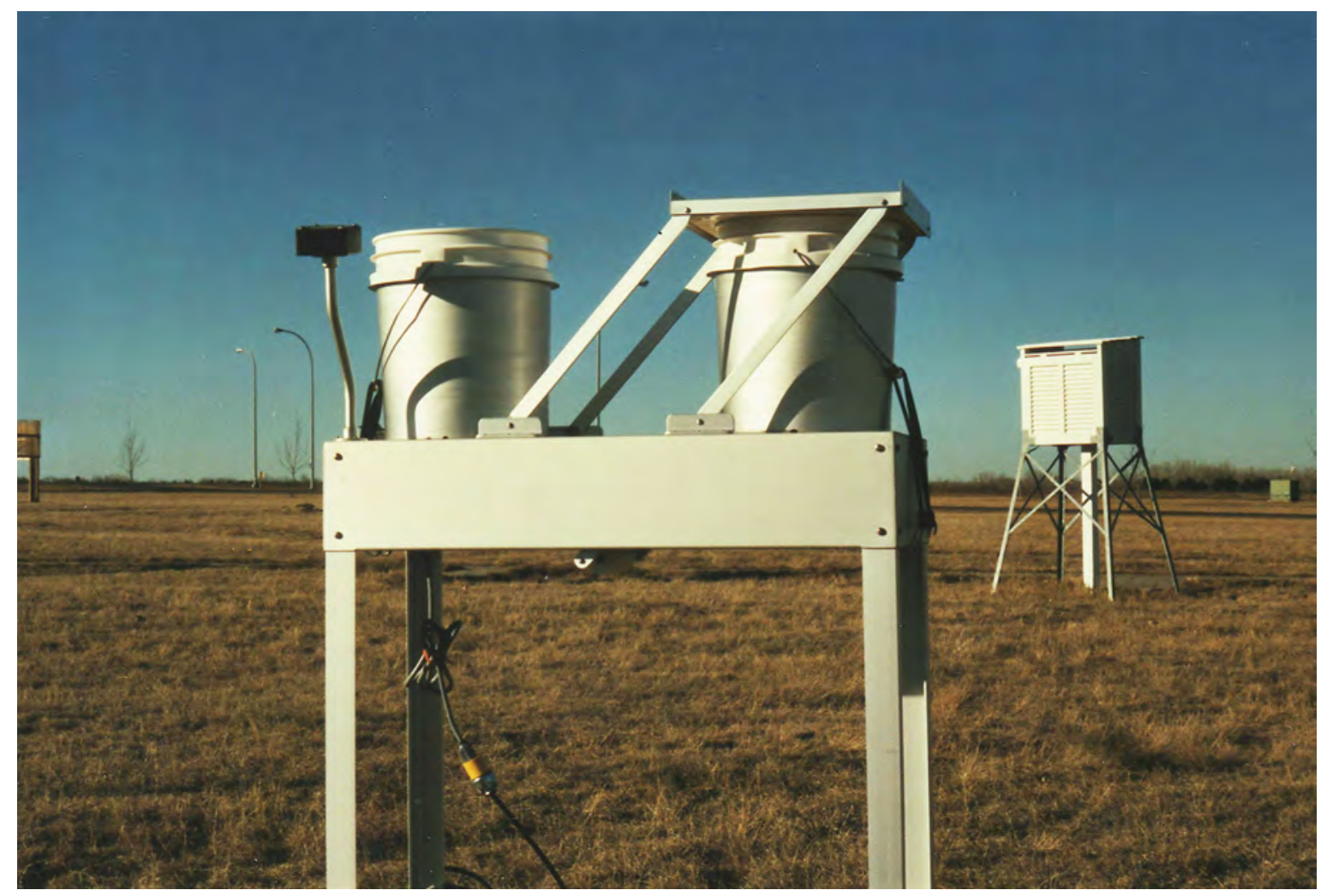

Figure 7. AeroChem Metrics (model 301) automatic sensing wet/dry precipitation collector.

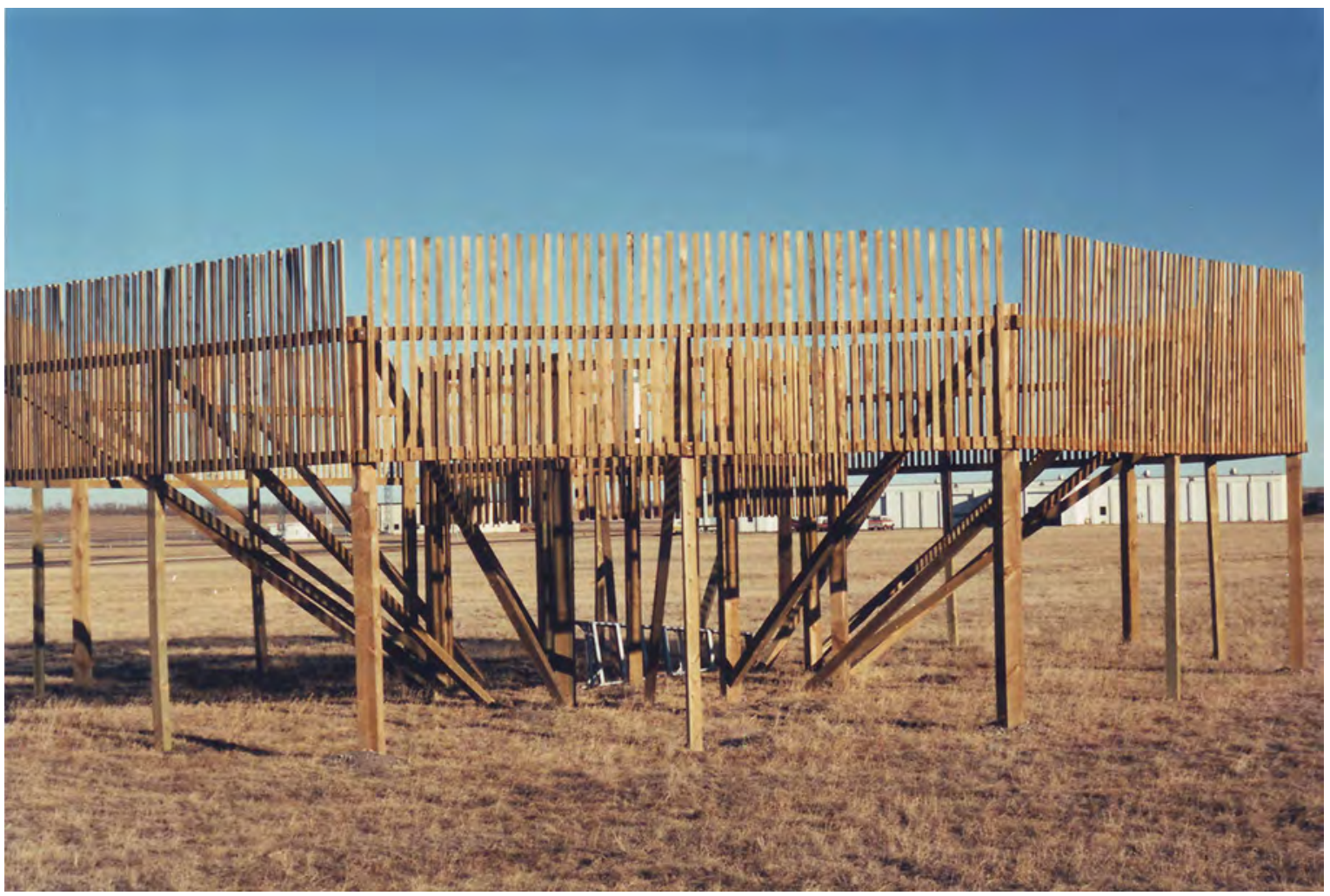

Figure 8. National (National Weather Service) gage with double fence shield. 


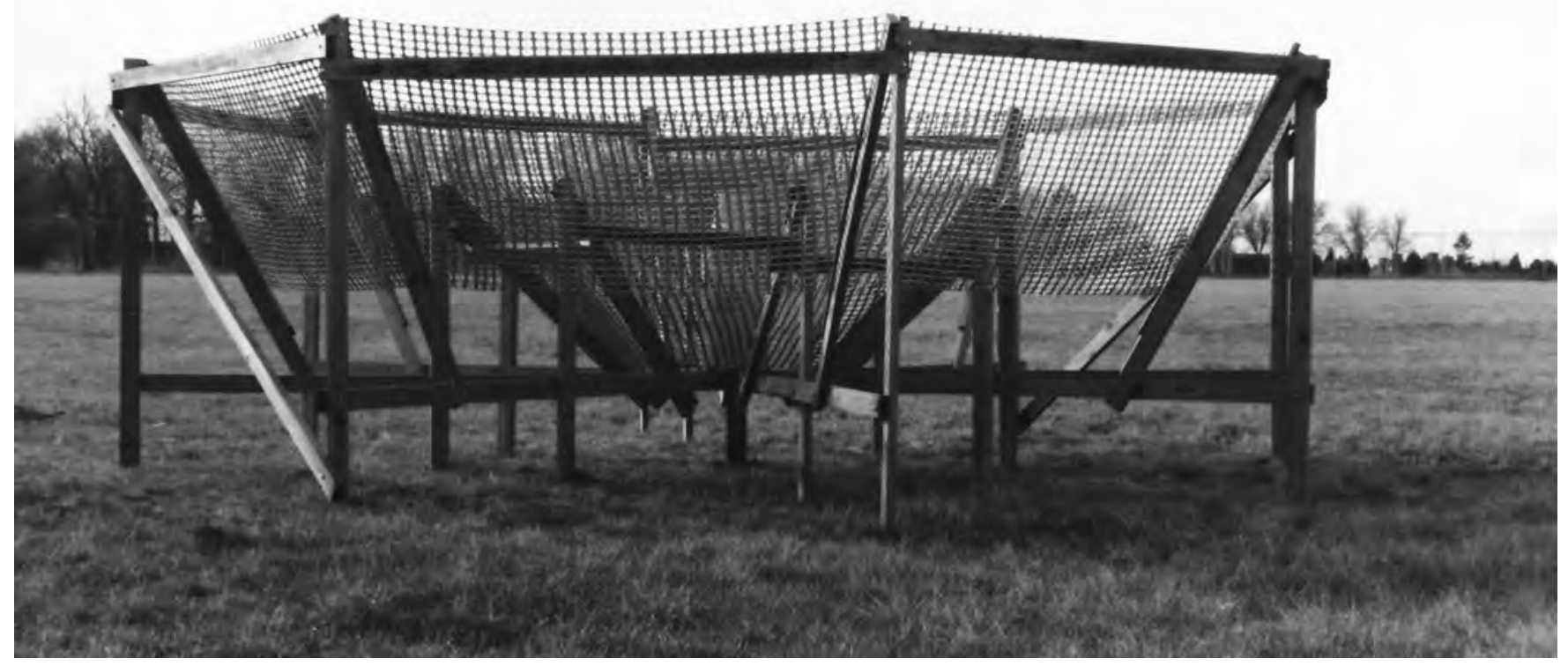

Figure 9. National (National Weather Service) gage with a Wyoming windshield.

\section{Methods}

The methods used to install and operate the solid precipitation measurement equipment and other meteorological observation equipment at the Bismarck station were those outlined by the WMO for the solid precipitation intercomparison (Goodison and others, 1998; Goodison and others, 1989). Data collection using the same methods continued after the official intercomparison ended.

\section{Solid Precipitation Event Data}

A solid precipitation event was defined as a period of time during which a discrete precipitation event occurred, resulting in precipitation accumulation in the DFIR of at least 3 millimeters (Goodison and others, 1998). The temperature data were determined from NWS hourly observations for the period of the event. Predominant wind direction was determined from hourly mean values recorded by the data logger at the wind direction sensor for the event. Mean event wind speed at the national standard height $(6.1 \mathrm{~m})$ was determined from NWS hourly observations for the period of the event. Mean event wind speeds at the orifice height of $1.4 \mathrm{~m}$ and at the orifice height of $3.0 \mathrm{~m}$ were determined from hourly mean values recorded by data loggers at the wind speed sensors for the event period (unpublished data; D.G. Emerson, U.S.
Geological Survey, written commun., 1990). The mean wind speed data at the national standard height $(6.1 \mathrm{~m})$ were not used in the analyses, but were published as part of the dataset for the intercomparison study.

Precipitation data for the event period were obtained from the manual gages (DFIR, Tretyakov, and AeroChem Metrics) and data logger recording gages (national standard gage with an Alter-type windshield, national standard gage without a windshield, national standard gage with double fence windshield, and national standard gage with a Wyoming windshield). Manual gages can have losses associated with wetting of devices used for the measurement (Goodison and others, 1998). Wetting losses were eliminated by using a laboratory digital balance to weigh the collection bucket of the manual gages to determine a gross weight, discarding the contents and letting the bucket dry completely, then weighing again to determine a tare weight. The gross weight minus the tare weight was the weight of the precipitation collected; this was then used to determine the precipitation.

In initial exploratory data analysis, an event that began on November 1, 1992, appeared to be a high outlier for a number of gages and had precipitation amounts ranging from 0 to 19.8 millimeters. No reason was identified for the great variation in measurement for this event, but data recording or data entry errors were suspected. Therefore, data from that event were removed from the dataset. The data used in this analysis are listed in table 1. 
Table 1. Data collected during solid precipitation measurement intercomparison in Bismarck, N. Dak., from 1988 through 1997.

[m, meters; DFIR, double fence intercomparison reference; adjusted, values adjusted by using equations 1 and 2 in this report; --, no data; R, rain; S, snow; M, mixed precipitation]

\begin{tabular}{|c|c|c|c|c|c|c|c|c|c|c|c|c|c|c|c|c|c|c|}
\hline \multicolumn{2}{|c|}{ Date and time } & \multicolumn{3}{|c|}{$\begin{array}{c}\text { Temperature } \\
\text { (degrees Celsius) }\end{array}$} & \multirow[b]{2}{*}{ 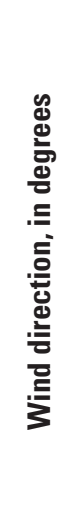 } & \multicolumn{3}{|c|}{$\begin{array}{c}\text { Wind speed } \\
\text { (meters per second) }\end{array}$} & \multicolumn{9}{|c|}{ Precipitation amount (millimeters) } & \multirow[b]{2}{*}{ 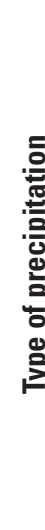 } \\
\hline 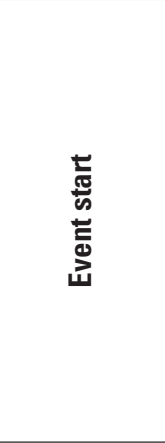 & 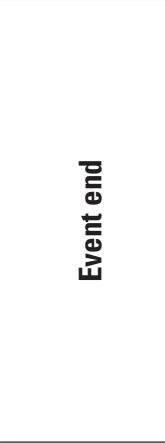 & $\begin{array}{l}\text { E } \\
\text { 亚 } \\
\text { 岕 }\end{array}$ & 㤩 & 惢 & & 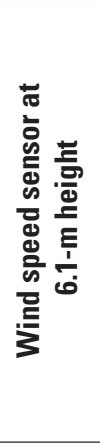 & 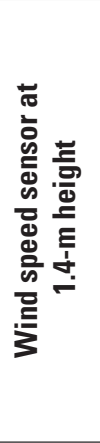 & 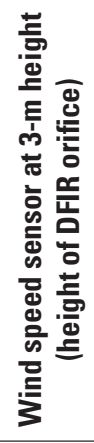 & 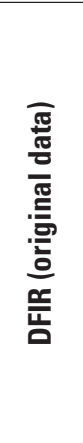 & 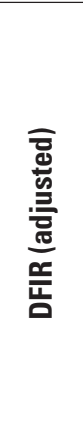 & 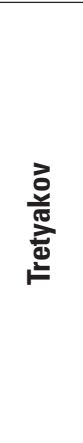 & 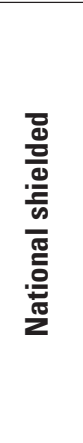 & 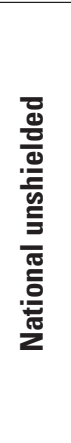 & 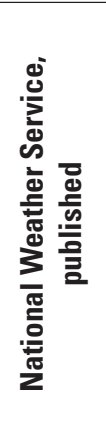 & 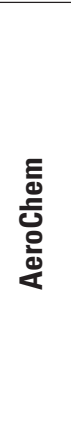 & 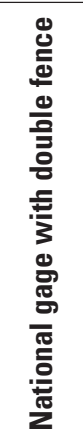 & 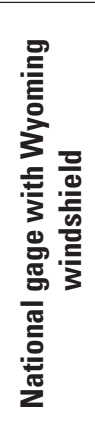 & \\
\hline $\begin{array}{l}11 / 06 / 1988 \\
1746\end{array}$ & $\begin{array}{l}11 / 06 / 1988 \\
2324\end{array}$ & 3.9 & 0.0 & 1.9 & 135 & 4.1 & 3.5 & 3.7 & 5.1 & -- & 4.6 & 3.3 & -- & 3.3 & 4.3 & 3.8 & -- & $\mathrm{R}$ \\
\hline $\begin{array}{l}11 / 14 / 1988 \\
1037\end{array}$ & $\begin{array}{l}11 / 15 / 1988 \\
0730\end{array}$ & -1.1 & -3.9 & -3.3 & 33 & 6.4 & 2.7 & 2.9 & 7.8 & 8.1 & 5.4 & 6.6 & -- & 6.6 & 2.2 & 7.1 & -- & $\mathrm{S}$ \\
\hline $\begin{array}{l}12 / 25 / 1988 \\
2040\end{array}$ & $\begin{array}{l}12 / 27 / 1988 \\
0930\end{array}$ & 1.7 & -3.3 & -.9 & 357 & 6.7 & 4.9 & 5.2 & 21.7 & 23.7 & 12.2 & 11.4 & -- & 11.7 & 1.0 & 20.0 & -- & $\mathrm{S}$ \\
\hline $\begin{array}{l}01 / 06 / 1989 \\
0100\end{array}$ & $\begin{array}{l}01 / 08 / 1989 \\
0710\end{array}$ & -14.3 & -17.6 & -15.9 & 25 & 7.9 & 6.1 & 6.7 & 14.3 & 16.3 & 10.1 & 8.6 & -- & 9.7 & .1 & 11.0 & -- & $\mathrm{S}$ \\
\hline $\begin{array}{l}02 / 25 / 1989 \\
0902\end{array}$ & $\begin{array}{l}02 / 27 / 1989 \\
1805\end{array}$ & -1.1 & -16.7 & -8.9 & 311 & 5.1 & 3.6 & 3.9 & 3.3 & 3.5 & 1.7 & 3.3 & -- & 3.3 & .9 & 3.7 & -- & $\mathrm{S}$ \\
\hline $\begin{array}{l}03 / 02 / 1989 \\
1410\end{array}$ & $\begin{array}{l}03 / 04 / 1989 \\
0315\end{array}$ & -15.7 & -19.2 & -17.4 & 36 & 5.9 & 4.4 & 4.7 & 7.5 & 8.1 & 3.3 & 3.4 & -- & 3.4 & .0 & 5.8 & -- & $\mathrm{S}$ \\
\hline $\begin{array}{l}03 / 13 / 1989 \\
1603\end{array}$ & $\begin{array}{l}03 / 14 / 1989 \\
0810\end{array}$ & -1.7 & -5.8 & -3.8 & 16 & 6.8 & 5.0 & 5.4 & 3.3 & 3.6 & 1.8 & 2.8 & -- & 2.8 & .1 & 2.3 & -- & $\mathrm{S}$ \\
\hline $\begin{array}{l}11 / 13 / 1989 \\
1941\end{array}$ & $\begin{array}{l}11 / 14 / 1989 \\
0335\end{array}$ & 1.1 & -4.4 & -1.6 & 86 & 2.6 & 2.2 & 2.6 & 4.4 & 4.5 & 4.3 & 3.8 & 0.3 & 3.8 & 3.9 & 2.0 & 1.8 & $\mathrm{~S}$ \\
\hline $\begin{array}{l}11 / 26 / 1989 \\
1920\end{array}$ & $\begin{array}{l}11 / 27 / 1989 \\
0915\end{array}$ & 3.9 & -10.6 & -3.4 & 12 & 8.2 & 6.0 & 6.9 & 4.0 & 4.6 & 1.9 & .8 & .0 & 0.8 & .2 & 0.5 & 0.3 & $\mathrm{~S}$ \\
\hline $\begin{array}{l}01 / 16 / 1990 \\
1045\end{array}$ & $\begin{array}{l}01 / 17 / 1990 \\
1845\end{array}$ & -1.1 & -16.7 & -8.9 & 11 & 3.1 & -- & -- & 8.3 & -- & 7.4 & 6.4 & 5.3 & 6.4 & 5.5 & 6.7 & 6.6 & $\mathrm{~S}$ \\
\hline $\begin{array}{l}02 / 15 / 1990 \\
0615\end{array}$ & $\begin{array}{l}02 / 16 / 1990 \\
0845\end{array}$ & -9.4 & -20.0 & -14.7 & 38 & 5.1 & 3.9 & 4.4 & 6.4 & 6.8 & 4.6 & 4.6 & 3.3 & 4.6 & .6 & 5.6 & 3.1 & $\mathrm{~S}$ \\
\hline $\begin{array}{l}03 / 14 / 1990 \\
0645\end{array}$ & $\begin{array}{l}03 / 15 / 1990 \\
2224\end{array}$ & 3.3 & -4.4 & -.5 & 308 & 6.7 & 3.4 & 4.0 & 6.0 & 6.3 & 5.0 & 4.1 & 2.4 & 3.8 & .7 & 5.4 & 5.3 & $\mathrm{~S}$ \\
\hline
\end{tabular}


Table 1. Data collected during solid precipitation measurement intercomparison in Bismarck, N. Dak., from 1988 through 1997.—Continued

[m, meters; DFIR, double fence intercomparison reference; adjusted, values adjusted by using equations 1 and 2 in this report; --, no data; R, rain; S, snow; M, mixed precipitation]

\begin{tabular}{|c|c|c|c|c|c|c|c|c|c|c|c|c|c|c|c|c|c|c|}
\hline \multicolumn{2}{|c|}{ Date and time } & \multicolumn{3}{|c|}{$\begin{array}{c}\text { Temperature } \\
\text { (degrees Celsius) }\end{array}$} & \multirow[b]{2}{*}{ 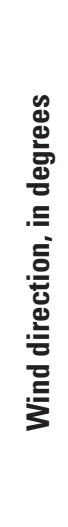 } & \multicolumn{3}{|c|}{$\begin{array}{c}\text { Wind speed } \\
\text { (meters per second) }\end{array}$} & \multicolumn{9}{|c|}{ Precipitation amount (millimeters) } & \multirow[b]{2}{*}{ 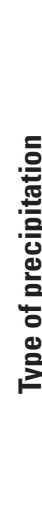 } \\
\hline 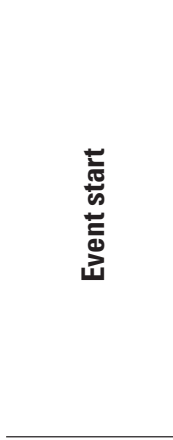 & 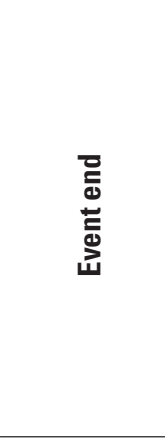 & 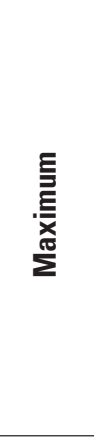 & $\begin{array}{l}\text { 豆 } \\
\text { 言 } \\
\text { 至 }\end{array}$ & 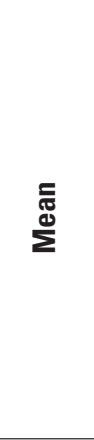 & & 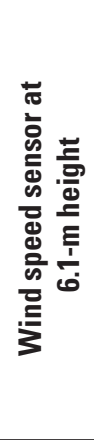 & 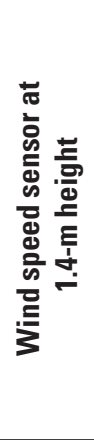 & 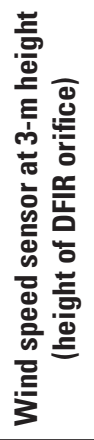 & 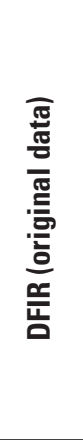 & 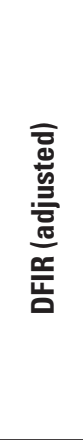 & 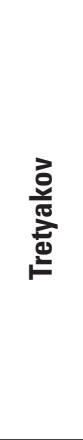 & 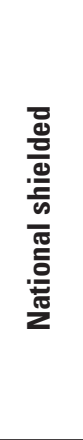 & 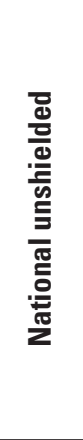 & 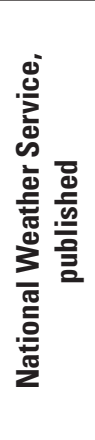 & $\begin{array}{l}\text { E } \\
\text { 덩 } \\
\text { 인 }\end{array}$ & 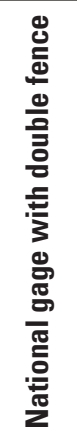 & 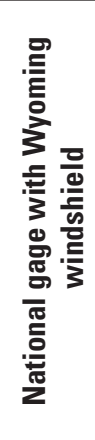 & \\
\hline $\begin{array}{l}12 / 02 / 1990 \\
0757\end{array}$ & $\begin{array}{l}12 / 03 / 1990 \\
1620\end{array}$ & -2.8 & -16.1 & -9.4 & 166 & 5.0 & 3.3 & 3.7 & 9.0 & 9.4 & 5.1 & 4.6 & 2.8 & 4.6 & 0.0 & 7.4 & 4.8 & $\mathrm{~S}$ \\
\hline $\begin{array}{l}03 / 12 / 1991 \\
0330\end{array}$ & $\begin{array}{l}03 / 13 / 1991 \\
1040\end{array}$ & 1.7 & -1.7 & .1 & 32 & 4.1 & 2.8 & 3.3 & 13.9 & 14.3 & 13.2 & 11.7 & 10.9 & 11.7 & 11.7 & 13.0 & 11.9 & M \\
\hline $\begin{array}{l}11 / 04 / 1991 \\
1200\end{array}$ & $\begin{array}{l}11 / 05 / 1991 \\
1413\end{array}$ & -2.6 & -9.3 & -5.9 & 292 & 6.7 & 4.6 & 5.1 & 4.3 & 4.7 & 3.6 & 3.0 & -- & 3.0 & 3.3 & 4.1 & 3.6 & $\mathrm{~S}$ \\
\hline $\begin{array}{l}11 / 17 / 1991 \\
1200\end{array}$ & $\begin{array}{l}11 / 18 / 1991 \\
0735\end{array}$ & 4.2 & 1.4 & 2.8 & 202 & 3.3 & 1.7 & 2.1 & 5.6 & -- & 15.8 & 8.6 & -- & 8.6 & 11.9 & 11.5 & 10.2 & $\mathrm{R}$ \\
\hline $\begin{array}{l}12 / 19 / 1991 \\
0001\end{array}$ & $\begin{array}{l}12 / 19 / 1991 \\
1631\end{array}$ & 1.7 & -1.4 & .1 & 158 & 6.9 & 3.6 & 4.3 & 3.8 & 3.9 & 3.2 & 3.1 & -- & 3.1 & 3.6 & 2.3 & -- & M \\
\hline $\begin{array}{l}01 / 14 / 1992 \\
0001\end{array}$ & $\begin{array}{l}01 / 14 / 1992 \\
1700\end{array}$ & -8.3 & -4.5 & -6.4 & 292 & 6.1 & 4.6 & 5.3 & 3.3 & 3.6 & 2.4 & 2.5 & -- & 2.5 & .5 & 3.5 & -- & $\mathrm{S}$ \\
\hline $\begin{array}{l}01 / 24 / 1992 \\
0001\end{array}$ & $\begin{array}{l}01 / 27 / 1992 \\
0845\end{array}$ & -6.2 & -12.9 & -9.5 & 90 & 4.3 & 2.8 & 3.2 & 8.9 & 9.2 & 5.3 & 4.8 & -- & 4.8 & .4 & 7.7 & -- & $\mathrm{S}$ \\
\hline $\begin{array}{l}02 / 10 / 1992 \\
1200\end{array}$ & $\begin{array}{l}02 / 11 / 1992 \\
1310\end{array}$ & -12.4 & -16.5 & -14.4 & 45 & 3.3 & 3.9 & 4.3 & 3.4 & 3.6 & 2.4 & 2.0 & -- & 2.0 & .0 & 1.5 & -- & $\mathrm{S}$ \\
\hline $\begin{array}{l}02 / 17 / 1992 \\
0001\end{array}$ & $\begin{array}{l}02 / 18 / 1992 \\
1238\end{array}$ & -1.3 & -2.6 & -1.9 & 315 & 4.0 & 3.2 & 3.5 & 7.7 & 7.9 & 6.8 & 6.1 & -- & 6.1 & 2.8 & 6.8 & -- & M \\
\hline $\begin{array}{l}03 / 05 / 1992 \\
0102\end{array}$ & $\begin{array}{l}03 / 07 / 1992 \\
1240\end{array}$ & 4.0 & 2.3 & 3.2 & 292 & 3.3 & 2.4 & 2.8 & 14.3 & -- & 12.1 & 9.9 & -- & 9.9 & 13.4 & 11.5 & -- & $\mathrm{R}$ \\
\hline $\begin{array}{l}03 / 20 / 1992 \\
0840\end{array}$ & $\begin{array}{l}03 / 21 / 1992 \\
0915\end{array}$ & -2.4 & -7.2 & -4.8 & 68 & 5.5 & 4.4 & 5.0 & 9.7 & 10.5 & 5.9 & 5.1 & -- & 5.1 & .1 & 7.9 & -- & $\mathrm{S}$ \\
\hline $\begin{array}{l}11 / 17 / 1992 \\
2020\end{array}$ & $\begin{array}{l}11 / 23 / 1992 \\
1120\end{array}$ & 1.0 & -.9 & .8 & 135 & 4.4 & 2.7 & 2.7 & 3.7 & 3.8 & 3.2 & 3.7 & 2.6 & 3.7 & 1.8 & 4.1 & 4.2 & $\mathrm{~S}$ \\
\hline
\end{tabular}


Table 1. Data collected during solid precipitation measurement intercomparison in Bismarck, N. Dak., from 1988 through 1997.—Continued

[m, meters; DFIR, double fence intercomparison reference; adjusted, values adjusted by using equations 1 and 2 in this report; --, no data; R, rain; S, snow; M, mixed precipitation]

\begin{tabular}{|c|c|c|c|c|c|c|c|c|c|c|c|c|c|c|c|c|c|c|}
\hline \multicolumn{2}{|c|}{ Date and time } & \multicolumn{3}{|c|}{$\begin{array}{c}\text { Temperature } \\
\text { (degrees Celsius) }\end{array}$} & \multirow[b]{2}{*}{ 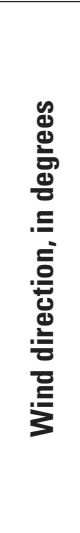 } & \multicolumn{3}{|c|}{$\begin{array}{c}\text { Wind speed } \\
\text { (meters per second) }\end{array}$} & \multicolumn{9}{|c|}{ Precipitation amount (millimeters) } & \multirow[b]{2}{*}{ 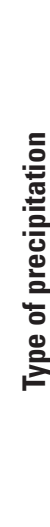 } \\
\hline 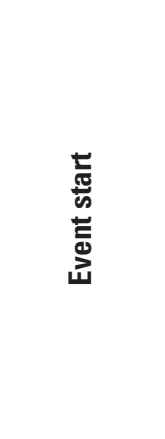 & 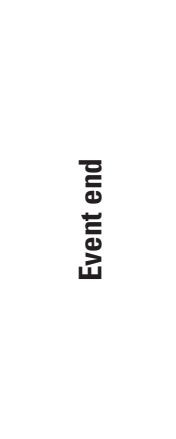 & $\begin{array}{l}\text { E } \\
\text { 皇 } \\
\text { 岕 }\end{array}$ & 志 & $\sum_{\Sigma}^{\bar{d}}$ & & 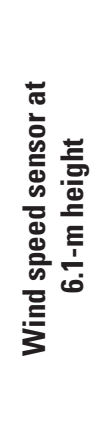 & 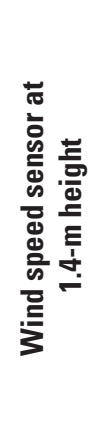 & 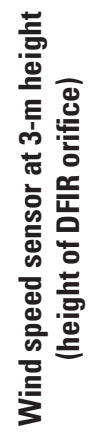 & 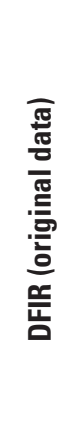 & 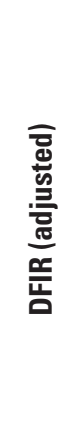 & 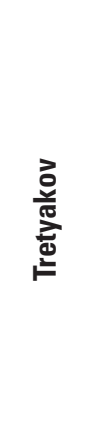 & 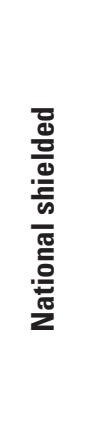 & 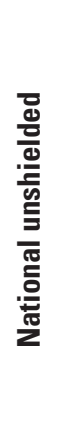 & 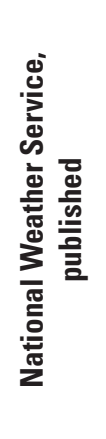 & 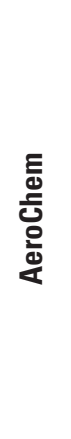 & 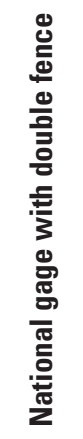 & 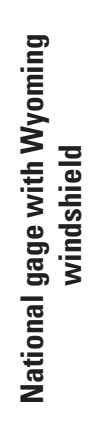 & \\
\hline $\begin{array}{l}12 / 12 / 1992 \\
1620\end{array}$ & $\begin{array}{l}12 / 13 / 1992 \\
1645\end{array}$ & -6.7 & -8.9 & -7.8 & 360 & 5.8 & 4.1 & 4.8 & 12.6 & 13.6 & 9.0 & 7.1 & 4.1 & 7.1 & 1.9 & 9.7 & 7.9 & $\mathrm{~S}$ \\
\hline $\begin{array}{l}01 / 11 / 1993 \\
1200\end{array}$ & $\begin{array}{l}01 / 14 / 1993 \\
0915\end{array}$ & -11.7 & -16.7 & -14.2 & 338 & 4.3 & -- & -- & 10.9 & -- & 6.6 & 4.8 & 4.1 & 4.8 & 1.2 & 9.1 & 6.1 & $\mathrm{~S}$ \\
\hline $\begin{array}{l}02 / 11 / 1993 \\
0335\end{array}$ & $\begin{array}{l}02 / 16 / 1993 \\
1730\end{array}$ & -11.9 & -18.4 & -15.1 & 338 & 3.3 & 2.2 & 2.5 & 4.7 & 4.8 & 3.3 & 3.6 & 3.3 & 3.6 & 1.9 & 3.8 & 2.5 & $\mathrm{~S}$ \\
\hline $\begin{array}{l}02 / 20 / 1993 \\
0001\end{array}$ & $\begin{array}{l}02 / 25 / 1993 \\
1015\end{array}$ & -14.7 & -21.7 & -18.2 & 360 & 2.7 & 2.1 & 2.4 & 7.7 & 7.9 & 6.1 & 4.8 & 3.3 & 4.8 & 2.6 & 5.9 & 4.6 & $\mathrm{~S}$ \\
\hline $\begin{array}{l}03 / 09 / 1993 \\
0225\end{array}$ & $\begin{array}{l}03 / 12 / 1993 \\
1530\end{array}$ & -.6 & -7.7 & -4.1 & 360 & 5.7 & 3.7 & 4.5 & 3.7 & 3.9 & 2.3 & 4.3 & 1.0 & 4.3 & -- & 1.3 & .0 & M \\
\hline $\begin{array}{l}03 / 29 / 1993 \\
1200\end{array}$ & $\begin{array}{l}03 / 31 / 1993 \\
2320\end{array}$ & 2.7 & .8 & 1.4 & 360 & 4.6 & 3.5 & 4.2 & 6.1 & 6.4 & 5.0 & 5.1 & .0 & 5.1 & -- & .0 & .0 & M \\
\hline $\begin{array}{l}11 / 23 / 1993 \\
1200\end{array}$ & $\begin{array}{l}11 / 27 / 1993 \\
0355\end{array}$ & -10.3 & -12.6 & -11.5 & 338 & 7.0 & 4.2 & 4.9 & 25.0 & 27.0 & 16.3 & 23.4 & -- & 23.4 & -- & -- & 26.7 & $\mathrm{~S}$ \\
\hline $\begin{array}{l}12 / 05 / 1993 \\
0535\end{array}$ & $\begin{array}{l}12 / 05 / 1993 \\
1335\end{array}$ & 2.8 & -1.7 & -.5 & 315 & 9.7 & 5.8 & 6.9 & 3.5 & 3.9 & 2.4 & 3.1 & 3.0 & 3.1 & -- & 3.8 & 3.3 & M \\
\hline $\begin{array}{l}12 / 16 / 1993 \\
0001\end{array}$ & $\begin{array}{l}12 / 17 / 1993 \\
2130\end{array}$ & -1.0 & -2.1 & -1.5 & 360 & 4.8 & 3.1 & 3.5 & 15.8 & 16.5 & 12.7 & 11.9 & 7.1 & 11.9 & -- & 14.0 & 11.4 & $\mathrm{~S}$ \\
\hline $\begin{array}{l}12 / 28 / 1993 \\
1843\end{array}$ & $\begin{array}{l}12 / 30 / 1993 \\
0338\end{array}$ & -6.1 & -14.2 & -10.1 & 292 & 6.9 & 4.1 & 4.6 & 5.9 & 6.3 & 4.2 & .8 & 1.8 & .8 & -- & 1.5 & .0 & $\mathrm{~S}$ \\
\hline $\begin{array}{l}01 / 01 / 1994 \\
1200\end{array}$ & $\begin{array}{l}01 / 08 / 1994 \\
1745\end{array}$ & -15.6 & -22.8 & -19.2 & 68 & 5.4 & 3.2 & 3.6 & 22.6 & 23.4 & 15.1 & 11.6 & 4.6 & 11.6 & -- & 17.7 & 13.4 & M \\
\hline $\begin{array}{l}02 / 06 / 1994 \\
0000\end{array}$ & $\begin{array}{l}02 / 12 / 1994 \\
1020\end{array}$ & -17.5 & -25.8 & -20.6 & 315 & 3.9 & 2.1 & 2.4 & 11.3 & 11.6 & 9.2 & 4.8 & 2.8 & 4.8 & -- & 4.8 & 4.6 & $\mathrm{~S}$ \\
\hline
\end{tabular}


Table 1. Data collected during solid precipitation measurement intercomparison in Bismarck, N. Dak., from 1988 through 1997. Continued

[m, meters; DFIR, double fence intercomparison reference; adjusted, values adjusted by using equations 1 and 2 in this report; --, no data; R, rain; S, snow; M, mixed precipitation]

\begin{tabular}{|c|c|c|c|c|c|c|c|c|c|c|c|c|c|c|c|c|c|c|}
\hline \multicolumn{2}{|c|}{ Date and time } & \multicolumn{3}{|c|}{$\begin{array}{c}\text { Temperature } \\
\text { (degrees Celsius) }\end{array}$} & \multirow[b]{2}{*}{ 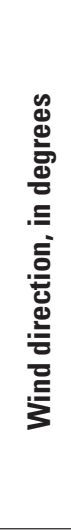 } & \multicolumn{3}{|c|}{$\begin{array}{c}\text { Wind speed } \\
\text { (meters per second) }\end{array}$} & \multicolumn{9}{|c|}{ Precipitation amount (millimeters) } & \multirow[b]{2}{*}{ 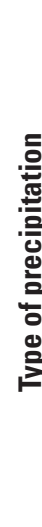 } \\
\hline 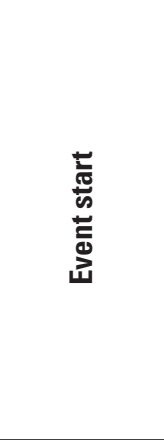 & 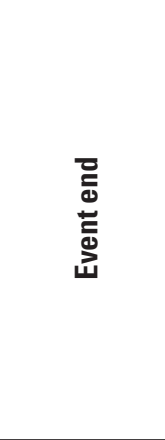 & $\begin{array}{l}\text { E } \\
\text { 恶 } \\
\text { 哥 }\end{array}$ & 竟 & స్ & & 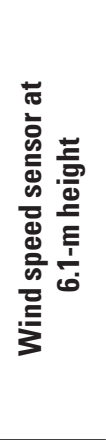 & 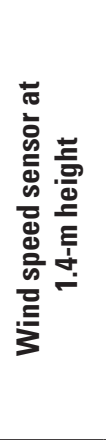 & 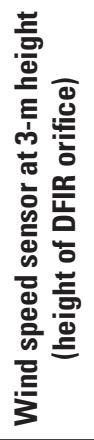 & 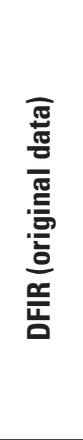 & 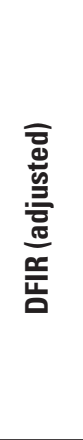 & 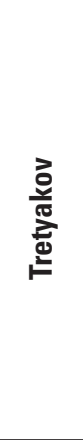 & 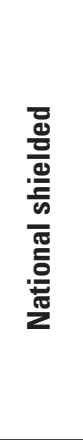 & 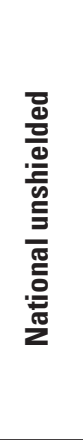 & 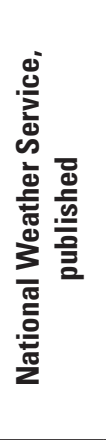 & 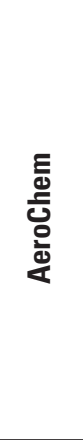 & 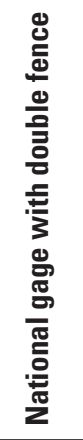 & 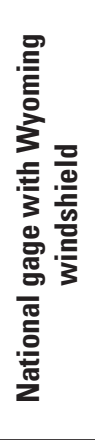 & \\
\hline $\begin{array}{l}02 / 18 / 1994 \\
1532\end{array}$ & $\begin{array}{l}02 / 23 / 1994 \\
0640\end{array}$ & -13.0 & -18.1 & -15.5 & 292 & 6.7 & 3.6 & 4.1 & 6.6 & 7.0 & 3.3 & 2.5 & 1.5 & 2.5 & -- & 4.8 & 3.8 & $\mathrm{~S}$ \\
\hline $\begin{array}{l}03 / 23 / 1994 \\
0041\end{array}$ & $\begin{array}{l}03 / 24 / 1994 \\
0558\end{array}$ & -1.7 & -8.5 & -5.1 & 360 & 5.3 & 3.7 & 4.1 & 13.8 & 14.6 & 8.0 & 9.4 & -- & 9.4 & -- & 4.1 & 2.3 & $\mathrm{~S}$ \\
\hline $\begin{array}{l}11 / 12 / 1994 \\
1443\end{array}$ & $\begin{array}{l}11 / 13 / 1994 \\
2326\end{array}$ & 8.0 & 4.3 & 6.1 & 292 & 6.4 & 4.1 & 4.4 & 10.1 & 10.6 & 10.0 & 8.6 & 11.4 & 8.6 & 9.8 & 10.7 & 9.1 & M \\
\hline $\begin{array}{l}11 / 17 / 1994 \\
1156\end{array}$ & $\begin{array}{l}11 / 18 / 1994 \\
1220\end{array}$ & .0 & -6.9 & -3.5 & 360 & 8.5 & 5.7 & 5.8 & 10.4 & 11.3 & 13.2 & 13.2 & 1.8 & 13.2 & 2.4 & 7.6 & 4.8 & $\mathrm{M}$ \\
\hline $\begin{array}{l}11 / 26 / 1994 \\
0124\end{array}$ & $\begin{array}{l}11 / 29 / 1994 \\
0122\end{array}$ & -3.7 & -8.2 & -5.9 & 292 & 6.6 & 4.4 & 4.6 & 16.0 & 16.9 & 12.8 & 16.3 & 6.1 & 16.3 & 5.4 & 15.2 & 9.9 & M \\
\hline $\begin{array}{l}12 / 13 / 1994 \\
1845\end{array}$ & $\begin{array}{l}12 / 15 / 1994 \\
1045\end{array}$ & -5.7 & -10.4 & -8.1 & 112 & 4.3 & 2.2 & 2.2 & 10.4 & 10.7 & 14.7 & 6.9 & 4.8 & 6.9 & 5.5 & 8.4 & 7.6 & $\mathrm{~S}$ \\
\hline $\begin{array}{l}01 / 13 / 1995 \\
1800\end{array}$ & $\begin{array}{l}01 / 17 / 1995 \\
1935\end{array}$ & -6.4 & -9.8 & -8.1 & 315 & 6.6 & 4.4 & 4.5 & 8.7 & 9.2 & 9.7 & 8.1 & 3.0 & 8.1 & 0.4 & 8.9 & 3.8 & $\mathrm{M}$ \\
\hline $\begin{array}{l}02 / 25 / 1995 \\
2335\end{array}$ & $\begin{array}{l}02 / 28 / 1995 \\
1415\end{array}$ & -7.6 & -15.8 & -11.7 & 315 & 4.1 & 3.0 & 3.2 & 7.3 & 7.5 & 4.8 & 5.6 & -- & 5.6 & 2.9 & 2.5 & 3.3 & $\mathrm{M}$ \\
\hline $\begin{array}{l}03 / 03 / 1995 \\
0924\end{array}$ & $\begin{array}{l}03 / 08 / 1995 \\
1150\end{array}$ & -14.6 & -22.8 & -18.7 & 338 & 4.6 & 3.5 & 3.6 & 7.0 & 7.3 & 4.8 & 8.6 & .0 & 8.6 & 1.0 & 8.1 & 4.1 & $\mathrm{~S}$ \\
\hline $\begin{array}{l}03 / 22 / 1995 \\
0052\end{array}$ & $\begin{array}{l}03 / 22 / 1995 \\
2301\end{array}$ & 4.7 & .3 & 2.5 & 90 & 4.4 & 2.7 & 2.8 & 20.8 & 21.3 & 19.6 & 19.3 & 13.2 & 19.3 & 20.4 & 10.2 & 15.0 & $\mathrm{M}$ \\
\hline $\begin{array}{l}03 / 26 / 1995 \\
0505\end{array}$ & $\begin{array}{l}03 / 28 / 1995 \\
0427\end{array}$ & 1.3 & -2.1 & -.4 & 338 & 6.9 & 4.6 & 4.6 & 16.6 & 17.6 & 10.9 & 15.0 & 6.1 & 15.0 & 6.5 & 13.0 & 8.1 & $\mathrm{M}$ \\
\hline $\begin{array}{l}11 / 05 / 1996 \\
1345\end{array}$ & $\begin{array}{l}11 / 06 / 1996 \\
1100\end{array}$ & 3.3 & -1.1 & .0 & 328 & 3.4 & 2.8 & 3.1 & 11.1 & 11.5 & 10.5 & 11.4 & 6.6 & 11.4 & 7.8 & 9.1 & -- & $\mathrm{S}$ \\
\hline
\end{tabular}


Table 1. Data collected during solid precipitation measurement intercomparison in Bismarck, N. Dak., from 1988 through 1997.—Continued

[m, meters; DFIR, double fence intercomparison reference; adjusted, values adjusted by using equations 1 and 2 in this report; --, no data; R, rain; S, snow; M, mixed precipitation]

\begin{tabular}{|c|c|c|c|c|c|c|c|c|c|c|c|c|c|c|c|c|c|c|}
\hline \multicolumn{2}{|c|}{ Date and time } & \multicolumn{3}{|c|}{$\begin{array}{c}\text { Temperature } \\
\text { (degrees Celsius) }\end{array}$} & \multirow[b]{2}{*}{ 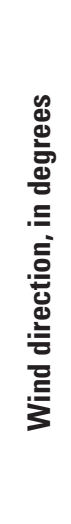 } & \multicolumn{3}{|c|}{$\begin{array}{c}\text { Wind speed } \\
\text { (meters per second) }\end{array}$} & \multicolumn{9}{|c|}{ Precipitation amount (millimeters) } & \multirow[b]{2}{*}{ 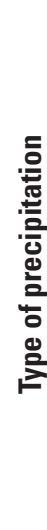 } \\
\hline 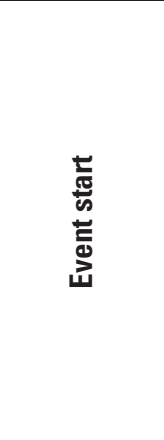 & 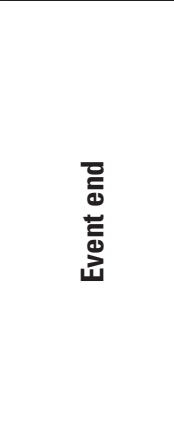 & 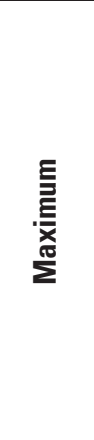 & 㤩 & 忢 & & 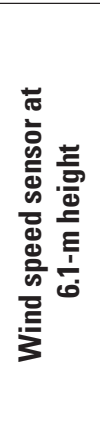 & 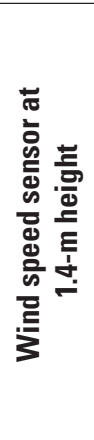 & 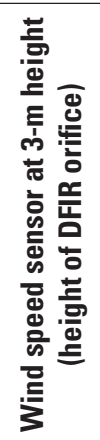 & 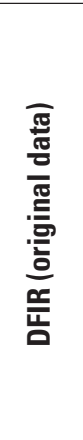 & 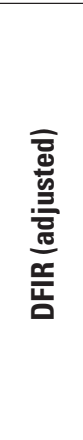 & 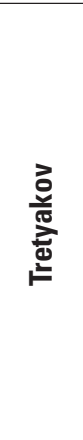 & 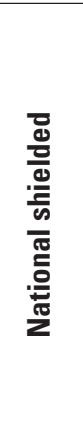 & 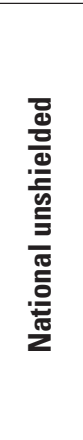 & 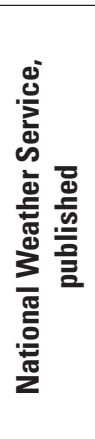 & 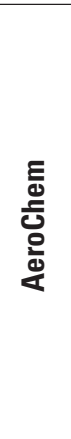 & 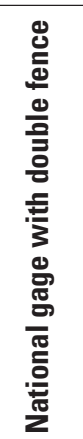 & 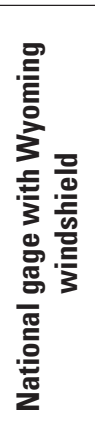 & \\
\hline $\begin{array}{l}11 / 15 / 1996 \\
2035\end{array}$ & $\begin{array}{l}11 / 16 / 1996 \\
0220\end{array}$ & -3.9 & -7.2 & -5.6 & 275 & 5.2 & 3.2 & 4.1 & 4.1 & 4.3 & 2.3 & 3.1 & 0.5 & 3.1 & 0.1 & 3.1 & 1.8 & $\mathrm{~S}$ \\
\hline $\begin{array}{l}11 / 19 / 1996 \\
0520\end{array}$ & $\begin{array}{l}11 / 20 / 1996 \\
1515\end{array}$ & -5.6 & -12.8 & -9.4 & 57 & 5.4 & 3.7 & 4.1 & 20.2 & 21.4 & 17.6 & 17.5 & 13.5 & 17.5 & 6.1 & 19.1 & 16.5 & $\mathrm{~S}$ \\
\hline $\begin{array}{l}11 / 23 / 1996 \\
0345\end{array}$ & $\begin{array}{l}11 / 24 / 1996 \\
0745\end{array}$ & -12.8 & -20.6 & -15.0 & 292 & 2.7 & 2.2 & 2.6 & 13.2 & 13.5 & 12.1 & 13.0 & 6.9 & 13.0 & 5.3 & 11.2 & 17.5 & $\mathrm{~S}$ \\
\hline $\begin{array}{l}01 / 04 / 1997 \\
0205\end{array}$ & $\begin{array}{l}01 / 05 / 1997 \\
0735\end{array}$ & -8.3 & -17.8 & -11.7 & 314 & 7.3 & 4.7 & 5.2 & 14.5 & 15.8 & 11.0 & 13.0 & 4.8 & 13.0 & 3.6 & 14.0 & 12.7 & $\mathrm{~S}$ \\
\hline $\begin{array}{l}01 / 23 / 1997 \\
1930\end{array}$ & $\begin{array}{l}01 / 26 / 1997 \\
0530\end{array}$ & -17.8 & -30.6 & -23.3 & 313 & 4.0 & 2.7 & 3.2 & 5.5 & 5.7 & 4.0 & 3.1 & -- & 3.1 & .5 & 3.3 & 2.0 & $\mathrm{~S}$ \\
\hline
\end{tabular}




\section{Correction of Data from the Double Fence Intercomparison Reference}

The DFIR is a secondary reference standard. The bush gage, a Tretyakov gage with windshield placed in a 12 -m-diameter working area with 2- to 4-m-high shrubs, is considered by WMO to be the primary standard because it is less adversely affected by wind speed than the DFIR (Yang and others, 1993). From a comparison of bush gage data to DFIR data at Valdai, Russia, Golubev (1989) found systematic differences and proposed an adjustment of the DFIR data that included meteorological measurements of wind speed, atmospheric pressure, air temperature, and humidity. Yang and others (1993) found that the most statistically significant factor in the adjustment of the DFIR data was the mean wind speed during the event. The sixth session of the International Organizing Committee of the WMO Solid Precipitation Measurement Intercomparison (World Meteorological Organization/ Commission for Instruments and Methods of Observation, 1993) recommended that the adjustment of Yang and others (1993) be applied to all DFIR data before analyzing the catch ratio (CR) of other gages with respect to that of the DFIR.

Yang and others (1993) proposed six equations for adjusting the DFIR data with respect to the bush gage data. The equations were for dry snow, wet snow, blowing snow, rain with snow, snow with rain, and rain, and all the equations were functions of wind speed. A reevaluation of the adjustment equations was recommended at the seventh session of the $\mathrm{WMO} / \mathrm{CIMO}$ because more intercomparison data were collected from the hydrological research station at Valdai, Russia (Goodison and others, 1998). The results of this reevaluation indicated that wind speed was the only statistically significant explanatory variable for the ratio of bush-to-DFIR catch and that wind speed was not statistically significant for rain. The equations of Yang and others (1993) were simplified to two regression equations for snow and mixed precipitation. For snow, the adjustment equation is

$$
\frac{B U S H}{D F I R}(\%)=100+(0.439 \times W S)+\left(0.246 \times W S^{2}\right),
$$

and for mixed precipitation the adjustment equation is

$$
\frac{B U S H}{D F I R}(\%)=100+(0.194 \times W S)+\left(0.222 \times W S^{2}\right),
$$

where

$$
\begin{gathered}
\text { BUSH is the snow or mixed precipitation catch, in } \\
\text { millimeters, at the bush gage; } \\
\text { is the snow or mixed precipitation catch, } \\
\text { in millimeters, at the double fence } \\
\text { intercomparison reference gage; and } \\
\text { is wind speed, in meters per second. }
\end{gathered}
$$

The final report of the WMO solid precipitation measurement intercomparison recommended that for future studies these two equations be used to adjust catch at the DFIR and that no adjustment be made for rain measurements of the DFIR (Goodison and others, 1998). Therefore, equations 1 and 2 were used to adjust the catch reported at the Bismarck DFIR gage for snow and mixed precipitation events. For two events, beginning January 16, 1990, and January 11, 1993, the wind speed at the DFIR gage was not recorded. Thus, precipitation for these events could not be adjusted and data from these events were not used in the remainder of the analysis.

\section{Correction of Catch Ratios}

Precipitation measurements can be affected by evaporation losses, by undercatch caused by wind, by precipitation type, and by the physics of the gage. Evaporation losses have been reported to be small for most gages (Golubev and others, 1992; Elomaa, 1993), and gage catch has not been corrected for evaporation loss in other studies (Yang and others, 1999); therefore, the data in this study were not corrected for evaporation loss.

For this study, events were categorized as snow, mixed, or rain on the basis of descriptive notes recorded as part of the intercomparison. The rain events data are published as part of this study but were not further analyzed (table 1). The $\mathrm{CRs}$ - the ratios of the precipitation catch at each gage to the true precipitation measurement (the adjusted double fence intercomparison reference) - were calculated for snow and mixed precipitation events by using the wind speed measured at the orifice height of each respective gage $(1.4 \mathrm{~m}$ or $3.0 \mathrm{~m})$. Regression analysis was used to develop equations that model the $\mathrm{CR}$ at each gage as functions of wind speed and temperature.

In previous studies, the results "show that not only wind speed but also air temperature affect the catch of some gauges, such as the Tretyakov" gage (Yang and Goodison, 1998, p. 6,224). "For the U.S. 8-inch standard nonrecording gauge, wind speed is the only factor with a statistically significant relationship with the gauge catch; air temperature does not have a statistically significant effect, when precipitation is classified into snow, rain, and mixed precipitation.** * Goodison found through a stepwise regression, that upper air (700 mbar) temperature had a positive correlation to the catch of Canadian Nipher snow gauge; this suggests that upper air temperature might be a better indicator of snow crystal characteristics during the storm. A similar assessment would be useful in assessing the catch of other gauges, such as the U.S. gauge" (Yang and Goodison, 1998, p. 6,224). Therefore, wind speed at the height of the gages, temperature (maximum, minimum, and mean), and upper air conditions [700 millibar (mbar) temperature and wind speed] were used as possible explanatory variables in the multiple regression analysis done for this study.

Upper air temperature and wind speed are collected by radiosonde and pilot balloon observations. As a radiosonde ascends into the atmosphere, measurements are made at 
mandatory pressure levels, including $700 \mathrm{mbar}$, and are transmitted to a ground receiving station (Durre and others, 2006). Upper air data for the Bismarck site were obtained from the National Oceanic and Atmospheric Administration Integrated Global Radiosonde Archive (IGRA) file transfer protocol site at $f t p: / / f t p . n c d c . n o a a . g o v / p u b /$ data/igra/. The IGRA data were collected at 12-hour intervals, which do not match perfectly with the beginning and end times of the precipitation events. For average upper air temperature and wind speed, the first observation immediately prior to the beginning of the precipitation event through the first observation immediately after the event were averaged. For upper air temperature and wind speed at the beginning of the precipitation event, the first observation immediately prior to the beginning of the precipitation event was used.

Figures 10 through 13 show the relations between gage CRs for the types of gages used for the Bismarck field study and possible explanatory variables to model the CRs. For snow events, wind speed affects some gages (AeroChem and the national unshielded) more than others (national with double fence), and the relation is not monotonic increasing or decreasing (as evidenced for the national with Wyoming windshield gage). The relation is even more complex for mixed precipitation events (fig. 10). The CRs for snow generally increase when mean, maximum, and minimum temperature increases, although it is not a simple relation (fig. 11). The same relations are highly varied for mixed precipitation (fig. 11). The relation between upper air temperature and CR varies with the gage and type of precipitation (fig. 12). Increased upper air wind speed at the beginning of an event decreases gage catch for snow, and the relation is more variable for mixed precipitation (fig. 13).

Many regression models can produce similar results; therefore, a procedure was used to develop a model in an unbiased manner. All-subset regression by leaps and bounds, a procedure that attempts to find the best regression relations by using subsets of the given explanatory variables (Insightful Corporation, 2005), was used to select the best regression model for estimating a particular CR. The possible explanatory variables were wind speed and powers of wind speed (powers of 1.25, 1.5, 1.75, 2, based on WMO equations); mean, maximum, and minimum temperature during event; the upper air temperature recorded immediately prior to event beginning; average upper air temperature recorded from the reading immediately prior to event beginning to immediately after event end; upper air wind speed recorded immediately prior to event beginning; and average upper air wind speed recorded from the reading immediately prior to event beginning to immediately after event end.
The criteria for determining the best regression relation were the adjusted coefficient of multiple determination $\left(\mathrm{R}_{a}^{2}\right)$ and Mallows' $\mathrm{C}_{p}\left(\mathrm{C}_{p}\right)$. The coefficient of determination $\left(\mathrm{R}^{2}\right)$, commonly used in model selection, increases with the number of explanatory variables in the regression model, but $\mathrm{R}_{a}^{2}$ allows for the comparison of models that have differing numbers of explanatory variables by penalizing models that have additional coefficients (Helsel and Hirsch, 1995). The $\mathrm{C}_{p}$ criterion is a measure of the total mean squared error and an indicator of model bias (Neter and others, 1996). In model comparison, the models with the smallest $\mathrm{C}_{p}$ values are considered to have the least bias.

In cases where several possible models were identified, exhaustive stepwise regression was performed with the potential variables identified in the all-subset regression by leaps and bounds. This automatic search procedure sequentially added terms to and deleted terms from the model used to determine the best subset of explanatory variables from a given set of variables (Insightful Corporation, 2005). The model chosen had a relatively small residual sum of squares, also called error sum of squares. In cases where multiple models were found to have similar residual sums of squares, the simpler model was chosen or, if the models were of the same size, the one with the smaller residual standard error and significant $\mathrm{p}$-value for the F-test was chosen. The F-test for regression relation tests whether there is a statistically significant regression relation between the $\mathrm{CR}$ and the set of explanatory variables in the regression model. The null hypothesis is that all regression coefficients are zero, and the alternative hypothesis is that at least one of the regression coefficients is not equal to zero. The existence of a regression relation supported by an F-test, however, does not ensure that the predictions based on the relation are useful (Neter and others, 1996).

As an indicator of the ability of the regression relations to estimate CRs, the observed CRs were compared with the CRs estimated by the regression equations by calculating relative percentage differences (RPDs) using the following equation:

$$
R P D=\left|\frac{E-D}{D} \times 100\right|,
$$

where

$$
\begin{aligned}
& E \quad \text { is the CR estimated from the regression } \\
& \text { equation, and } \\
& D \quad \text { is the CR from the data. }
\end{aligned}
$$

$D$ is assumed to be correct and the $R P D$ is the relative percentage difference of $E$ from $D$. 

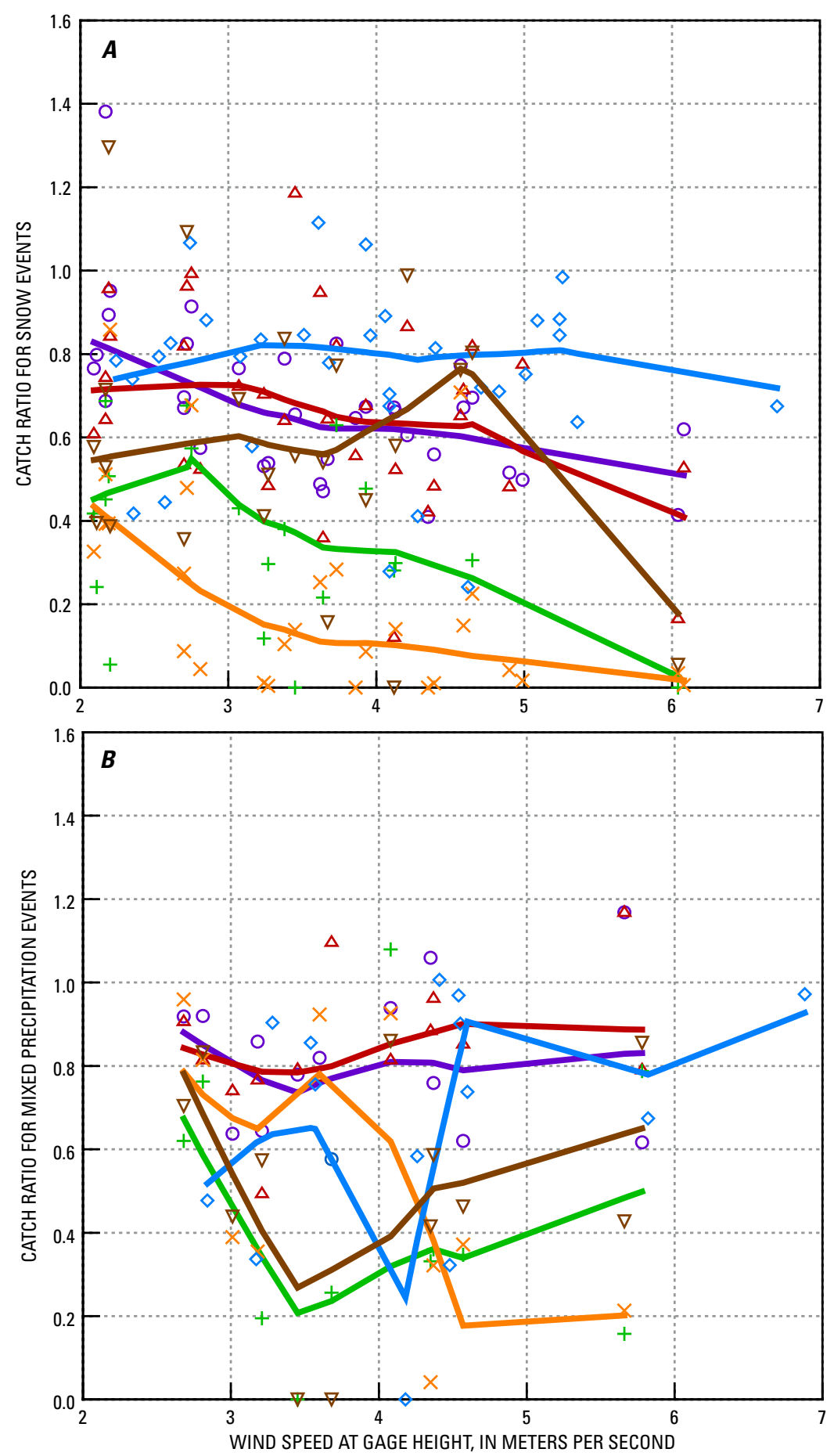

EXPLANATION

RATIO AND WIND SPEED

FOR A SPECIFIC EVENT

0
$\Delta$
+
$x$
$\diamond$
$\nabla$
LOWESS SMOOTH OF EVENTS

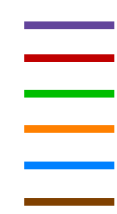

GAGE

Tretyakov

National shielded

National unshielded

AeroChem

National with double fence

National with Wyoming fence

Figure 10. Ratios of gage catch to the corrected double fence intercomparison reference and wind speeds for events in Bismarck, N. Dak., from 1988 through 1997. A, Snow; B, Mixed precipitation. 

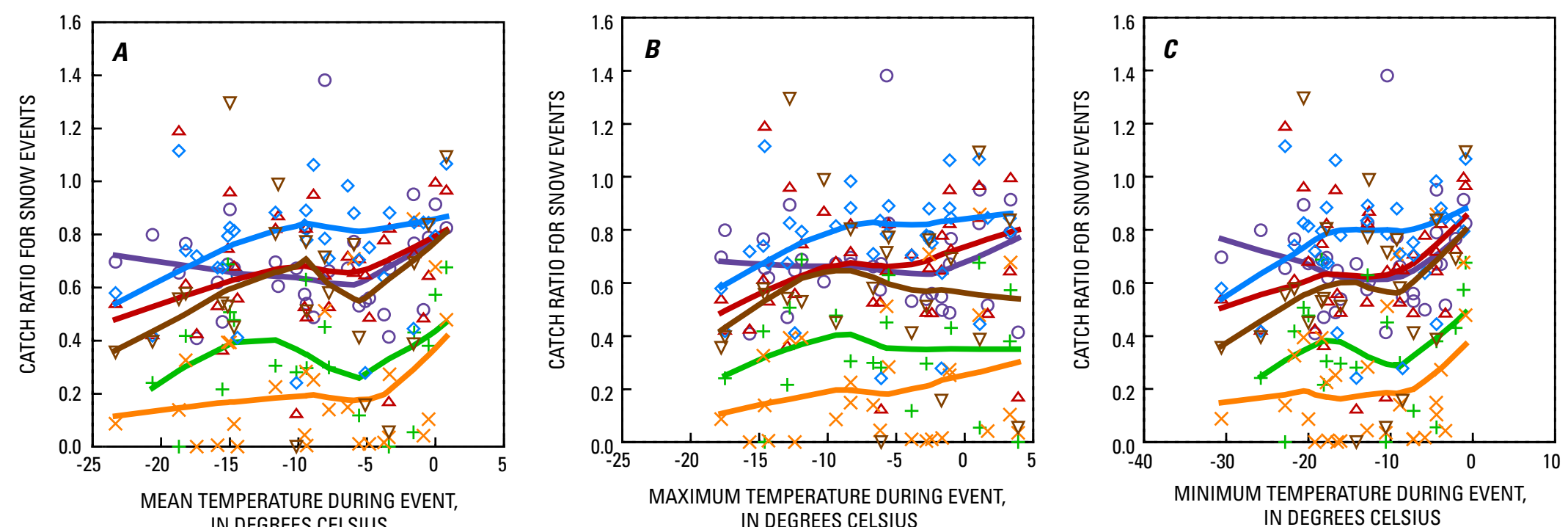

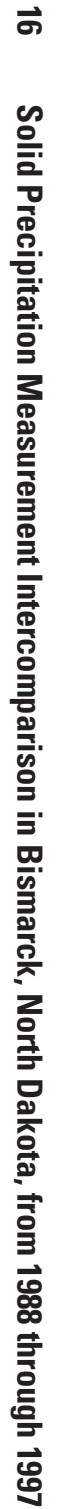
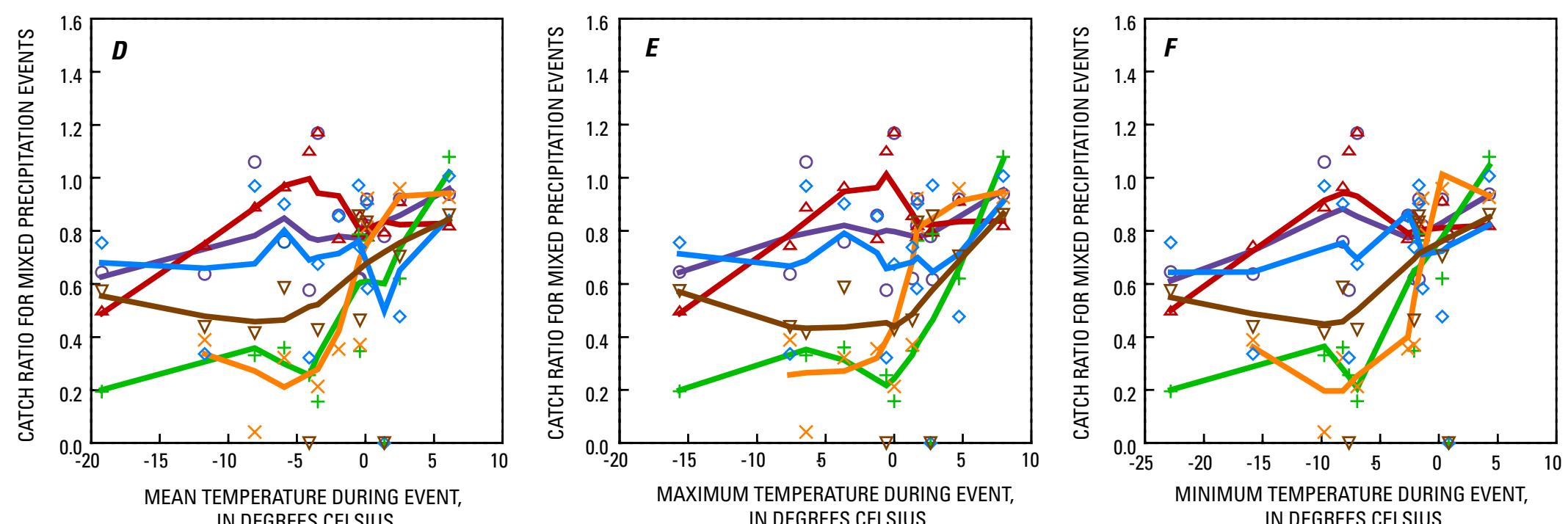

RATIO AND WIND SPEED FOR A SPECIFIC EVENT

EXPLANATION

LOWESS SMOOTH

GAGE OF EVENTS

Figure 11. Ratios of gage catch to the corrected double fence

intercomparison reference and mean, maximum, and minimum

temperatures for events in Bismarck, N. Dak., from 1988 through

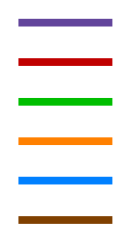

Tretyakov

National shielded

National unshielded

AeroChem

National with double fence

National with Wyoming fence 

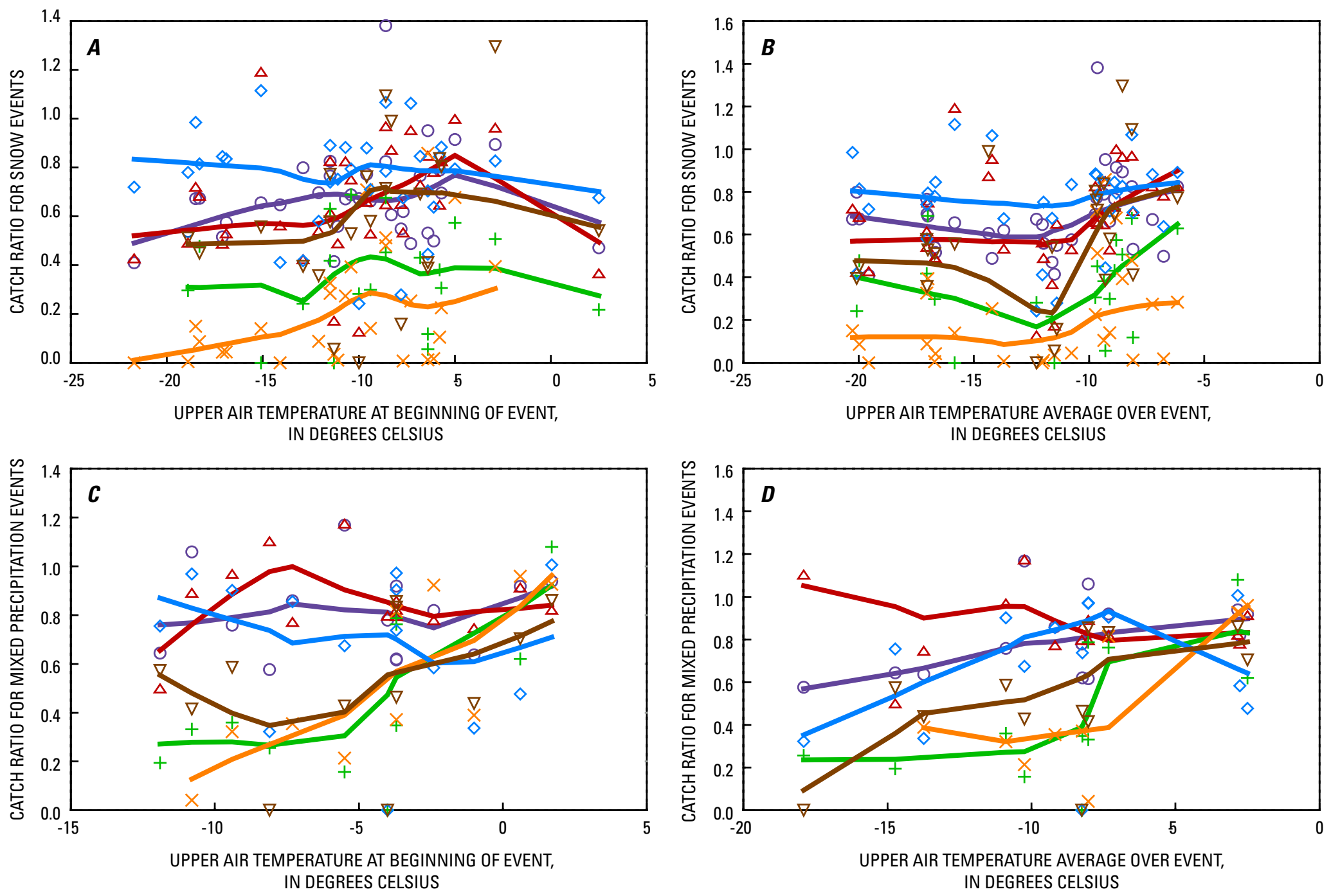

IN DEGREES CELSIUS

EXPLANATION

\begin{tabular}{ccl} 
RATIO AND WIND SPEED & LOWESS SMOOTH & \multicolumn{1}{l}{ GAGE } \\
FOR A SPECIFIC EVENT & OF EVENTS & \\
0 & & Tretyakov \\
$\Delta$ & & National shielded \\
+ & & National unshielded \\
$\times$ & & AeroChem \\
$\diamond$ & & National with double fence \\
$\nabla$ & & National with Wyoming fence
\end{tabular}

in Bismarck, N. Dak., from 1988 through 1997.

$A$, At the beginning of snow events; $B$, Averages for snow

events; $C$, At the beginning of mixed precipitation events;

$D$, Averages for mixed precipitation events.

National with Wyoming fence 

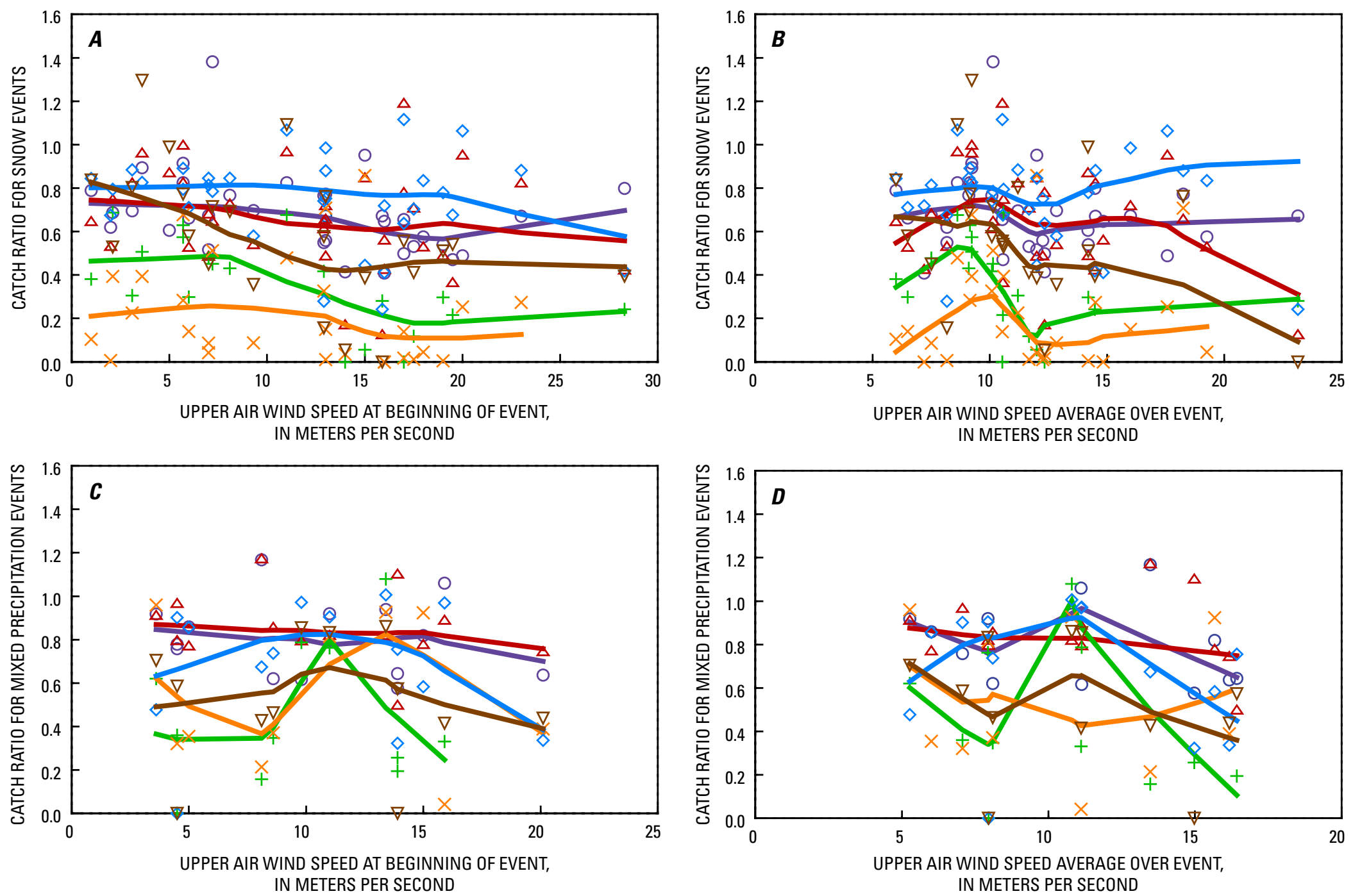

IN METERS PER SECOND

RATIO AND WIND SPEED FOR A SPECIFIC EVENT

$\begin{array}{ll}\circ & \\ \Delta & \\ + & \\ \times & \\ \diamond & \\ \nabla & \end{array}$

Figure 13. Ratios of gage catch to the corrected double fence intercomparison reference and upper air (700-millibar pressure) wind speed in Bismarck, N. Dak., from 1988 through 1997. A, At the beginning of snow events; $B$, Averages for snow events; $C$, At the beginning of mixed precipitation events; $D$, Averages for mixed precipitation events.

\section{EXPLANATION}

DWESS SMOOTH

GAGE

Tretyakov

National shielded

National unshielded

AeroChem

National with double fence

National with Wyoming fence 


\section{Solid Precipitation Measurement Intercomparison}

In addition to the regression equations, the $\mathrm{CR}$ can also be expressed as a ratio:

$$
C R=\frac{P_{g}}{P_{D F I R}},
$$

where

$$
\begin{gathered}
P_{g} \quad \begin{array}{c}
\text { is the precipitation measured at a particular } \\
\text { gage, in millimeters; and }
\end{array} \\
P_{D F I R} \quad \begin{array}{c}
\text { is the precipitation measured and corrected at } \\
\text { the reference gage, in millimeters. }
\end{array}
\end{gathered}
$$

This ratio can also be used to adjust the catch at a gage to what the catch would be at the DFIR using the formula $P_{D F I R}=P_{g} / C R$. The efficacy of this adjustment depends on the equation for $C R$ and the efficacy of the adjustments was examined for snow and mixed precipitation.

\section{Snow Precipitation}

The CRs were modeled by using multiple regression analysis for the Tretyakov gage, national shielded gage, national unshielded gage, AeroChem gage, national gage with double fence, and national gage with Wyoming windshield. The models were chosen by using the procedure described in the "Methods" section and evaluated by using $\mathrm{R}^{2}, \mathrm{R}_{a}^{2}$, standard error, and the F-test for regression relation. The results are listed in table 2 .

As in earlier studies, wind speed and air temperature were found to influence the CR for the Tretyakov gage. However, in this instance, the temperature variable represented the average upper air temperature over the duration of the event. The WMO did not use upper air conditions in its analysis. Wind speed and wind speed squared are both in the model for this study. The WMO CR equation used wind speed squared only. However, this study used the hierarchical approach to fitting a polynomial regression model. If a polynomial term is used in the model, all lower order terms are retained in the model as well because the lower order term provides more basic information about the response, whereas the higher order term provides refinement in the response function (Neter and others, 1996).

The national shielded and unshielded gages were found to be influenced by functions of wind speed only, as in other studies. However, the upper air wind speed was used, and again a hierarchical approach to fitting was used in which, if wind speed squared was used in the model, wind speed was retained in the model as well.

The AeroChem gage was not used in the WMO intercomparison study for 1987-93. It had a highly varied CR (table 1), and a number of variables related to wind speed and temperature were used in the model for the CR.

Despite extensive efforts to find a model for the national gage with double fence, no statistically significant regression model was found at the 0.05 level $(\alpha$-level). (A model was considered significant if the $\mathrm{p}$-value for the F-test of regression relation was less than 0.05.) Table 2 shows the best model found had a p-value 0.078 for the F-test. This model used wind speed terms to model the $\mathrm{CR}$ and had a relatively small $\mathrm{R}^{2}$ of 0.206 . This gage had one data value that appeared to be an outlier, a small CR for the event that began November 26, 1989 (table 1); however, there was no reason to remove this observation from the analysis, and removal did not improve the results.

The national gage with Wyoming windshield had a CR modeled by temperature and wind speed variables. It had the largest $\mathrm{R}^{2}$ and $\mathrm{R}_{a}^{2}$ of all of the models identified, 0.572 and 0.476 (table 2).

Three of the gage CRs evaluated could be compared with those in the WMO intercomparison (table 3), which had the advantage of a much larger dataset. However, the data in this study represent a longer time period. The differences in $\mathrm{R}^{2}$ and standard error largely may be because of the differences in sample size. The relations between the $\mathrm{CR}$ regression equations are shown in figure 14. If the regression equations adjusted the catch perfectly, the points would all fall on the diagonal line - the line of equality for observed and modeled catch. The red triangles are the catch predicted by using the equations developed in this study and converting from CR to catch (CR equals the catch at a gage divided by the catch at the DFIR; therefore, catch at a gage equals the CR for that gage multiplied by the catch at the DFIR). The red line is a lowess scatter plot smooth line (Insightful Corporation, 2005) used to show the general relation between the observed catch and the catch predicted by the equations developed in this study. If the relation was perfectly modeled, the lowess line would fall on the diagonal line. The blue diamonds are the catch predicted by the WMO CR equations and conversion from CR to catch. The blue line is a lowess scatter plot smooth line used to show the general relation between the observed catch and catch predicted by the WMO equations. Points below and to the right of the diagonal line underestimate the catch. Points above and to the left of the line overestimate the catch. The RPDs, which are indicators of the ability of the regression relations to estimate precipitation catch, are also shown in figure 14.

The RPDs for all three gages are smaller with the use of equations developed in this study than with the use of the WMO equations. For the DFIR Tretyakov gage (fig. 14A), both the WMO equations and the equations from this study do relatively well in predicting precipitation in the lower range. The upper range becomes more varied, particularly for the WMO equations, which tend to overcorrect the DFIR Tretyakov precipitation. The national shielded gage (fig. 14B) is similar in that variation appears to increase with snow amount. The equation from this study predicts values closer to the line of equality than does the WMO equation; however, both tend 
Table 2. Results of regression analysis of solid precipitation measurement intercomparison data from Bismarck, N. Dak., 1998 through 1997.

[n, number of samples used to develop regression equations; $\mathrm{R}^{2}$, coefficient of multiple determination; $\mathrm{R}_{a}^{2}$, adjusted coefficient of multiple determination; SE, standard error; $\mathrm{p}$-value for $\mathrm{F}$ test of significant regression relation; $C R$, catch ratio; $W S$, wind speed; $W_{i n d S p d}$, upper air wind speed at beginning of event; Tmp 700 , upper air temperature averaged over event; $T$, mean temperature; $T$, maximum temperature; $T_{\min }$, minimum temperature]

\begin{tabular}{|c|c|c|c|c|c|c|}
\hline Gage & Snow catch ratio equation & $\mathbf{n}$ & $\mathbf{R}^{2}$ & $\mathbf{R}^{2}$ & SE & $\begin{array}{l}\text { p-value for } \\
\text { F test }\end{array}$ \\
\hline Tretyakov & $C R=165.81-(31.47 \times W S)+\left(2.73 \times W S^{2}\right)-\left(0.98 \times\right.$ WindSpd $\left._{B}\right)+\left(0.88 \times \operatorname{Tmp}^{\prime} 700_{A v e}\right)$ & 34 & 0.564 & 0.504 & 13.1 & 0.000 \\
\hline National shielded & $C R=63.41+(16.90 \times W S)-\left(3.29 \times W S^{2}\right)-\left(1.02 \times W_{i n d S p d}\right)$ & 34 & .231 & .154 & 21.2 & .046 \\
\hline National unshielded & $C R=62.70+(3.30 \times W S)-\left(1.62 \times W S^{2}\right)-\left(1.77 \times W_{i n d S p d}\right)$ & 20 & .548 & .463 & 15.4 & .004 \\
\hline AeroChem & $C R=134.05-(39.68 \times W S)+\left(3.60 \times W S^{2}\right)+\left(1.37 \times T_{\text {mean }}\right)-\left(0.63 \times\right.$ WindSpd $\left._{B}\right)$ & 28 & .459 & .365 & 19.2 & .005 \\
\hline National gage with double fence & $C R=-22.15+(47.56 \times W S)-\left(4.71 \times W S^{2}\right)-\left(1.17 \times W_{i n d S p d}\right)$ & 33 & .206 & .124 & 21.9 & .078 \\
\hline $\begin{array}{l}\text { National gage with Wyoming } \\
\text { windshield }\end{array}$ & $C R=98.19-\left(17.49 \times T_{\max }\right)-\left(10.05 \times T_{\min }\right)+\left(27.49 \times T_{\text {mean }}\right)-\left(1.86 \times\right.$ WindSpd $\left._{B}\right)$ & 23 & .572 & .476 & 22.5 & .003 \\
\hline
\end{tabular}

Table 3. Solid precipitation measurement intercomparison data from Bismarck, N. Dak., 1988 through 1997, adjusted by regression analysis and World Metrological Organization solid precipitation measurement intercomparison equations.

[Source: Goodison and others, 1998. n, numbers of samples used to develop regression equations; $\mathrm{R}^{2}$, coefficient of multiple determination; SE, standard error; RPD, relative percentage difference; WMO, World Meteorological Organization; $C R$, catch ratio; WS, wind speed; $W_{i n d S p d}$, upper air wind speed at beginning of event; $T m p 700_{A v e}$, upper air temperature averaged over event; $T_{m a x}$, maximum temperature]

\begin{tabular}{|c|c|c|c|c|c|}
\hline Gage & Snow catch ratio equation & $\mathbf{n}$ & $\mathbf{R}^{2}$ & SE & RPD \\
\hline \multicolumn{6}{|l|}{ Tretyakov: } \\
\hline Regression analysis & $C R=165.81-(31.47 \times W S)+\left(2.72 \times W S^{2}\right)-\left(0.98 \times W_{i n d S p d}\right)+\left(0.88 \times \operatorname{Tmp}_{B} 700_{A v e}\right)$ & 34 & 0.564 & 13.1 & 9.6 \\
\hline WMO intercomparison & $C R=103.11-(8.67 \times W S)+\left(0.30 \times T_{\max }\right)$ & 241 & .400 & 11.1 & 14.4 \\
\hline \multicolumn{6}{|l|}{ National shielded: } \\
\hline Regression analysis & $C R=63.41+(16.90 \times W S)-\left(3.29 \times W S^{2}\right)-\left(1.02 \times W_{i n d S p d}\right)$ & 34 & .231 & 21.2 & 19.9 \\
\hline WMO intercomparison & $C R=\exp \left(4.61-\left(0.04 \times W S^{1.75}\right)\right)$ & 107 & .720 & 9.8 & 25.1 \\
\hline \multicolumn{6}{|l|}{ National unshielded: } \\
\hline Regression analysis & $C R=62.70+(3.30 \times W S)-\left(1.62 \times W S^{2}\right)-\left(1.77 \times W i n d S p d_{B}\right)$ & 20 & .548 & 15.4 & 26.9 \\
\hline WMO intercomparison & $C R=\exp \left(4.61-\left(0.16 \times W S^{1.28}\right)\right)$ & 55 & .770 & 9.4 & 28.1 \\
\hline
\end{tabular}



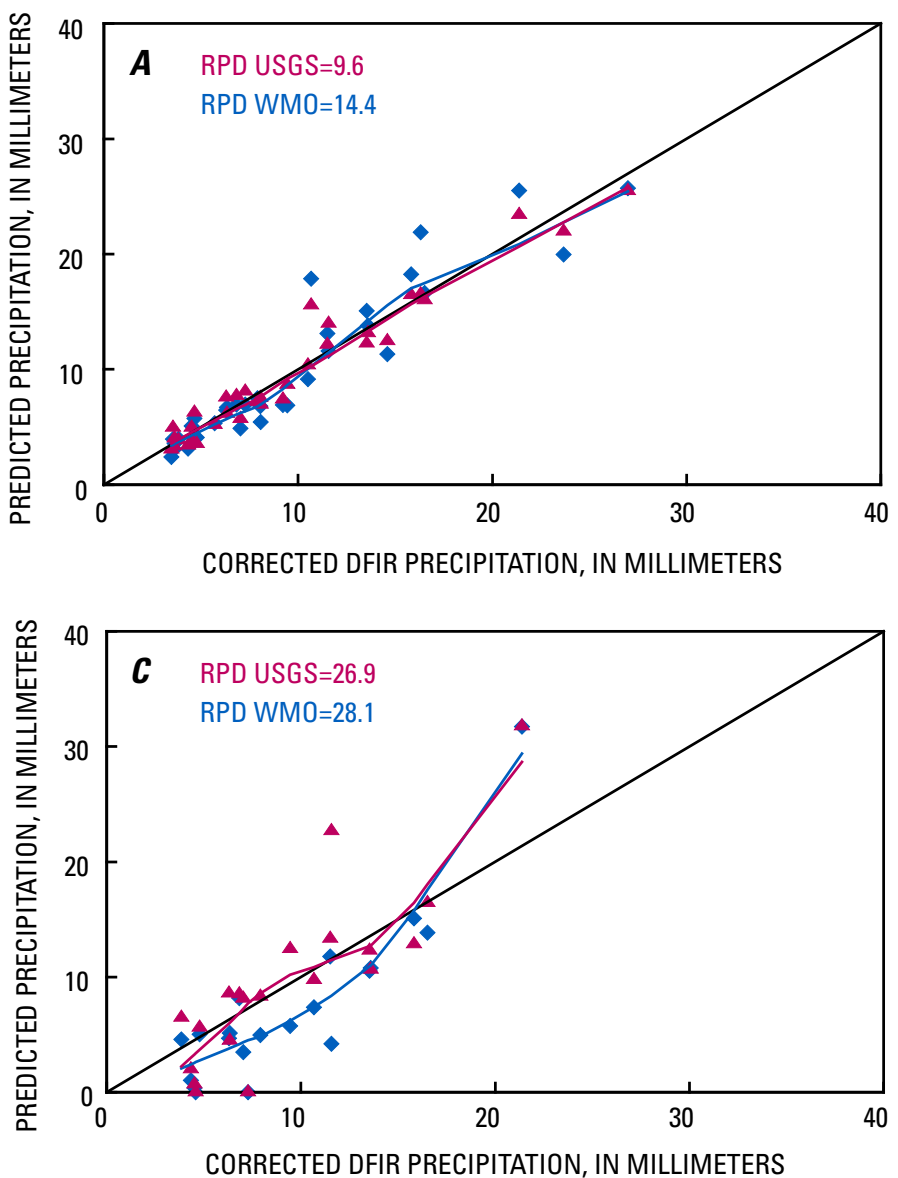

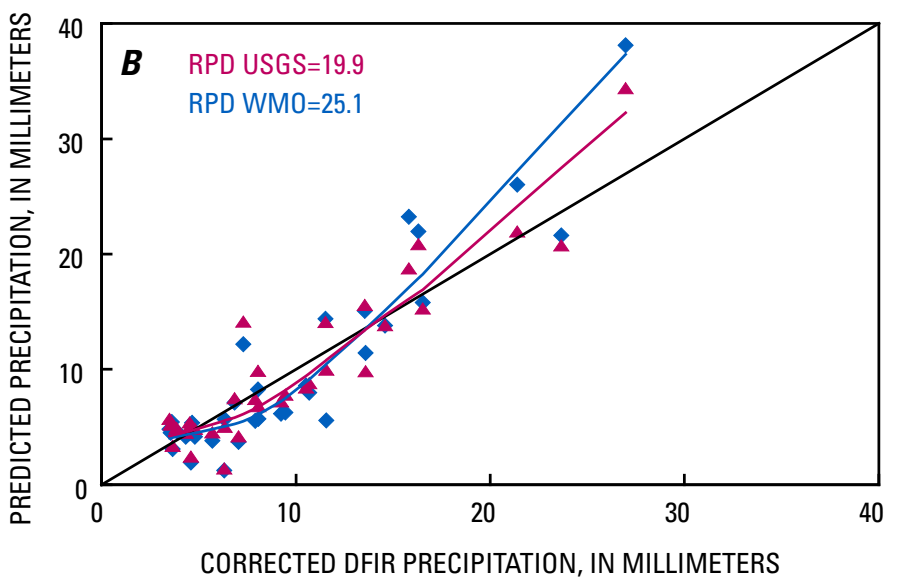

EXPLANATION

— Line of equality for corrected DFIR and modeled catch

- Lowess smooth line for precipitation catch predicted by equations in this study

- Lowess smooth line for precipitation catch predicted by the WMO equations

- Precipitation catch predicted by equations in this study

- Precipitation catch predicted by the WMO equations

RPD Median relative percentage difference between the catch ratio observed at a gage and the catch ratio estimated by the regression equations

Figure 14. Predicted snow catch based on World Meteorological Organization (WMO) equations and equations developed in this study (USGS), and corrected reference gage [double fence intercomparison reference (DFIR)] snow catch for three gages. $A$, Tretyakov; $B$, national shielded; $C$, national unshielded.

to underestimate in the low range and overestimate in the high range of precipitation values. The national unshielded gage (fig. 14C) has the largest RPDs for snow measurement, which is reasonable considering the gage is unshielded and greatly affected by meteorological conditions.

Snow (solid precipitation) catch is highly varied depending on the equipment used and the weather conditions. Much of the variation is not accounted for in the WMO equations or in the equations developed in this study, particularly for unshielded gages.

\section{Mixed Precipitation}

Despite extensive attempts at regression analysis with the mixed precipitation data, it was concluded that the sample sizes were not large enough for modeling the CRs (table 4). However, the data could be used to test the WMO equations. Figure 15 shows graphical relations between the catch at the DFIR and the predicted catch at the gages based on the WMO equations. The RPDs reflect the comparison of CRs modeled by the WMO equations (table 5) to the $\mathrm{CR}$ of the intercomparison reference standard, DFIR.

Table 4. Number of mixed precipitation observations at each gage at Bismarck, N. Dak., from 1988 through 1997.

[n, number of samples]

\begin{tabular}{lc}
\hline \multicolumn{1}{c}{ Gage } & n \\
\hline Tretyakov & 14 \\
National shielded & 14 \\
National unshielded & 11 \\
AeroChem & 10 \\
National gage with double fence & 14 \\
National gage with Wyoming windshield & 12 \\
\hline
\end{tabular}



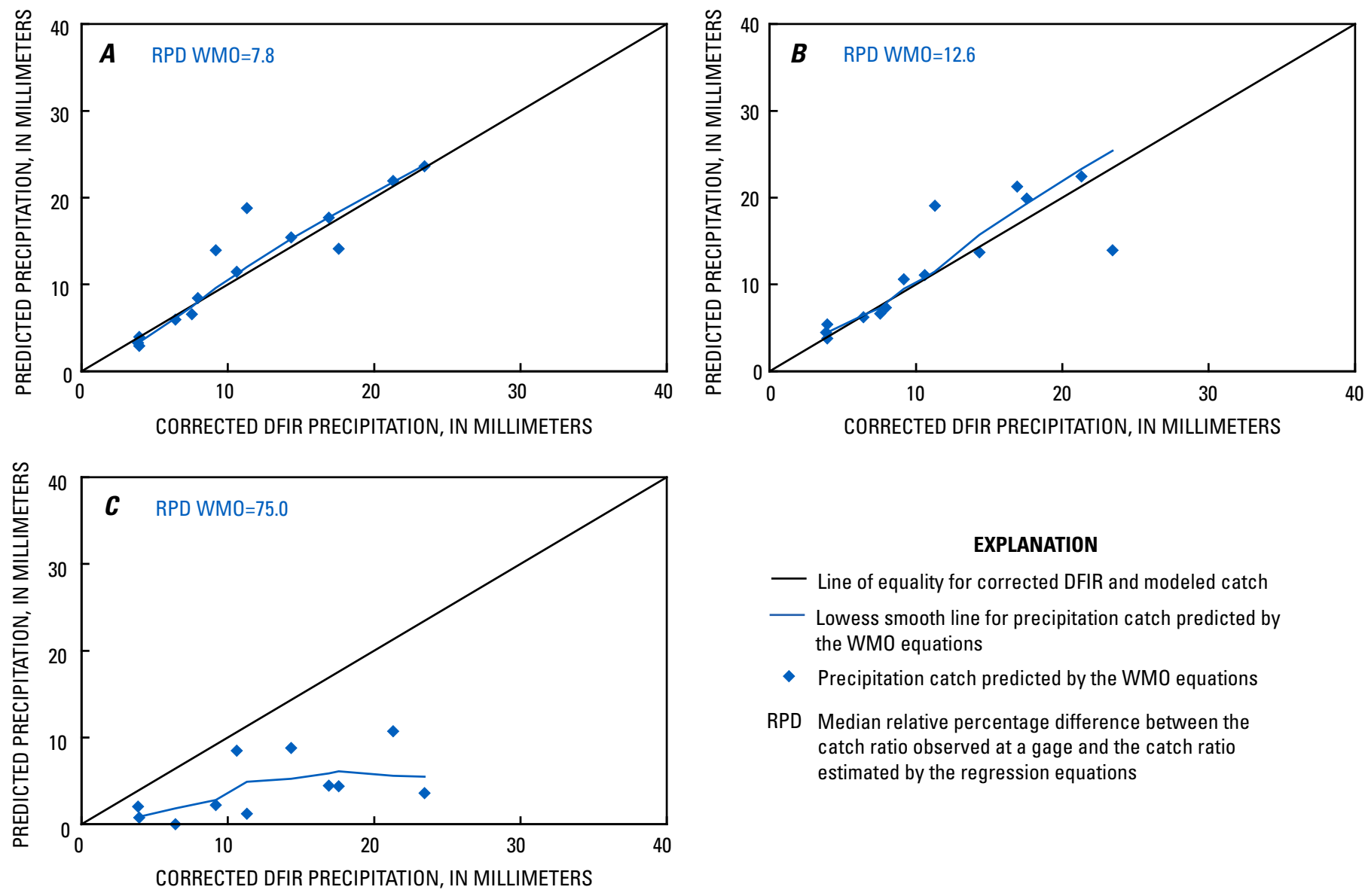

\section{EXPLANATION}

- Line of equality for corrected DFIR and modeled catch

- Lowess smooth line for precipitation catch predicted by the WMO equations

- Precipitation catch predicted by the WMO equations

RPD Median relative percentage difference between the catch ratio observed at a gage and the catch ratio estimated by the regression equations

Figure 15. Predicted mixed precipitation catch based on World Meteorological Organization (WMO) equations and corrected reference gage [double fence intercomparison reference (DFIR)] mixed precipitation catch for three gages. $A$, Tretyakov; $B$, national shielded; $C$, national unshielded.

Table 5. Catch ratio equations for mixed precipitation from the World Meteorological Organization solid precipitation measurement intercomparison.

[Source: Goodison and others, 1998. n, number of samples used to develop regression equations; $\mathrm{R}^{2}$, coefficient of multiple determination; SE, standard error; $\mathrm{RPD}$, relative percentage difference; $C R$, catch ratio; $W S$, wind speed; $T_{\max }$, maximum temperature; $T_{\min }$, minimum temperature]

\begin{tabular}{llrrrr}
\hline \multicolumn{1}{c}{ Gage } & \multicolumn{1}{c}{ Equation } & \multicolumn{1}{c}{$\mathbf{n}$} & \multicolumn{1}{c}{$\mathbf{R}^{2}$} & \multicolumn{1}{c}{ SE } & RPD \\
\hline Tretyakov & $C R=96.99-(4.46 \times W S)+\left(0.88 \times T_{\max }\right)+\left(0.22 \times T_{\text {min }}\right)$ & 433 & 0.460 & 8.0 & 7.8 \\
National shielded & $C R=101.04-(5.62 \times W S)$ & 75 & .590 & 7.6 & 12.6 \\
National unshielded & $C R=100.77-(8.34 \times W S)$ & 59 & .370 & 13.7 & 75.0 \\
\hline
\end{tabular}


The equations for mixed precipitation at the Tretyakov and national shielded gages are similar to those for snow in that they are more likely to underestimate precipitation in the low range and overestimate amounts in the high range (figs. 14 and 15). Mixed precipitation is greatly underestimated by the WMO adjustment and the national unshielded gage (RPD of 75.0).

\section{Summary and Conclusions}

A solid precipitation measurement intercomparison was recommended by the WMO (World Meteorological Organization) and was initiated after approval the ninth session of the Commission for Instruments and Methods of Observation (CIMO-IX). The goal of the intercomparison was to assess national methods of measuring solid precipitation against methods whose accuracy and reliability were known. A field study was started in Bismarck during the 1988-89 winter as part of the intercomparison. The last official field season of the WMO intercomparison was 1992-93. The Bismarck site continued to operate through the winter of 1996-97.

The intercomparison was designed to

1. determine wind related errors in national methods of measuring solid precipitation,

2. derive standard methods for adjusting solid precipitation measurements, and

3. introduce a reference method of solid precipitation measurement for general use to calibrate any type of precipitation gage.

The intercomparison found that discrepancies of as much as 110 percent existed among snowfall records from various national gages. On the basis of the intercomparison results, regression equations were developed to model the catch ratios (CRs) of the various solid precipitation instrumentation.

The study conducted in Bismarck did not use the Hellmann or Canadian Nipher gages, which were used at some intercomparison sites; however, it included additional gages: an AeroChem Metrics gage, a National Weather Service (NWS; national) gage with a double fence, and a national gage with a Wyoming windshield. Instrumentation at the Bismarck site included a double fence intercomparison reference (DFIR) having a Tretyakov gage (the reference gage for the WMO intercomparison and this study), a Tretyakov precipitation gage, a national gage equipped with the national standard windshield, a national gage without windshield, an NWS temperature and humidity sensing system, wind speed and wind direction sensors, an AeroChem Metrics (model 301) automatic sensing wet and dry precipitation collector, a double fence shield having a national gage installed in the center, and a national gage with a Wyoming windshield.

The DFIR gage catch for snow and mixed precipitation events was adjusted to what the catch would have been for a bush gage (a Tretyakov gage with windshield placed in an area surrounded by shrubs), which is considered by WMO to be the primary standard because it is less adversely affected by wind speed than the DFIR. The correction equations used were those recommended by the final report of the WMO solid precipitation measurement intercomparison for future studies.

Events at Bismarck were categorized as snow, mixed, or rain on the basis of descriptive notes recorded as part of the intercomparison. The rain events data were not further analyzed in this study. The CRs were calculated. Then, regression analysis was used to develop equations that model the snow and mixed precipitation CRs at each gage as functions of wind speed and temperature. Wind speed at the gages, functions of temperature, and upper air conditions were used as possible explanatory variables in the multiple regression analysis done for this study. The models were chosen using a all-subset regression approach and evaluated by using $\mathrm{R}^{2}$ (coefficient of multiple determination), $\mathrm{R}_{a}^{2}$ (adjusted coefficient of multiple determination), standard error, and the F-test for regression relation.

As in earlier studies, wind speed and air temperature were found to influence the CR for the Tretyakov gage. However, the temperature variable represented the average upper air temperature over the duration of the event. The WMO did not use upper air conditions in their analysis; however, use of upper air conditions had been suggested for future analyses.

The national shielded and unshielded gages where found to be influenced by functions of wind speed only, as in other studies. However, the upper air wind speed was used in the regression equations developed in this study.

The AeroChem gage was not used in the WMO intercomparison study for 1987-93. It had a highly varied CR at Bismarck, and a number of variables related to wind speed and temperature were used in the model for the CR. Despite extensive efforts to find a model for the national gage with double fence, no statistically significant regression model was found at the 0.05 level.

The national gage with Wyoming windshield had a CR modeled by temperature and wind speed variables. It had the largest $\mathrm{R}^{2}$ and $\mathrm{R}^{2}$ of all of the models identified, 0.572 and 0.476 .

Three of the gage CRs evaluated could be compared with those in the WMO intercomparison, which had the advantage of a much larger dataset. However, the data is this study represented a longer time period. The effectiveness of the $\mathrm{CR}$ regression equations were compared numerically and graphically.

Extensive attempts at regression analysis were made with the mixed precipitation data, but it was concluded that the sample sizes were not large enough for modeling the CRs. However, the data could be used to test the WMO equations. The equations for mixed precipitation at the Tretyakov and national shielded gages are similar to those for snow in that they are more likely to underestimate precipitation in the low range and overestimate amounts in the high range. Mixed 
precipitation is underestimated by the WMO adjustment for the national unshielded gage.

Results show that the precision of snow and mixed precipitation measurement varies greatly depending on the equipment used and the weather conditions. Mixed precipitation catch is highly varied, and both mixed and snow catch is highly varied for unshielded gages.

\section{References}

Durre, Imke, Vose, R.S., and Wuertz, D.B., 2006, Overview of the Integrated Global Radiosonde Archive: Journal of Climate, v. 19, no. 1, January 1, 2006, p. 53-68.

Elomaa, E.J., 1993, Experiences in correcting point precipitation measurement in Finland, in Proceedings of Eighth Symposium on Meteorological Observation and Instrumentation, Anaheim, Calif., January 17-22, 1993: Boston, Mass., American Meteorological Society, p. 346-350.

Golubev, V.S., 1989, Assessment of accuracy characteristics of the reference precipitation gauge with a double-fence shelter, in Final report of the Fourth Session of the International Organizing Committee for the WMO Solid Precipitation Measurement Intercomparison: Geneva, Switzerland, World Meteorological Organization, p. 22-29.

Golubev, V.S., Groisman, P.Y., and Quayle, R.G., 1992, An evaluation of the United States standard 8-inch nonrecording raingage at the Valdai polygon, Russia: Journal of Atmospheric and Oceanic Technology, v. 9, no. 5, October, p. 624-629.

Goodison, B.E., Louie, P.Y.T., and Yang, D., 1998, WMO solid precipitation measurement intercomparison-Final report: World Meteorological Organization Instruments and Observing Methods Report No. 67, WMO/TD-No. 872, 318 p., accessed August 23, 2008, at http://www.wmo.ch/ pages/prog/www/IMOP/publications/IOM-67-solid-precip/ WMOtd872.pdf

Goodison, B.E., Sevruk, B., and Klemm, S., 1989, WMO solid precipitation measurement intercomparison-objectives, methodology, analysis, in Delleur, J.W., ed., Atmospheric deposition [Proceedings of the Third Scientific Assembly, Baltimore, Md., May 1989]: International Association of Hydrological Sciences Publication No. 179, p. 57-64.

Helsel, D.R., and Hirsch, R.M., 1995, Statistical methods in water resources: New York, Elsevier Science B.V., 529 p.

Insightful Corporation, 2005, S-PLUS language reference, in S-PLUS 7.0 for Windows professional developer: Seattle, Insightful Corporation [variously paged].
Neter, J., Kutner, M.H., Nachtsheim, C.J., and Wasserman, W., 1996, Applied linear statistical models (4th ed.): Boston, Mass., WCB/McGraw-Hill, 1,408 p.

Yang, Daqing, and Goodison, B.E., 1998, Comment on "Reducing biases in estimates of precipitation over the United States: Phase 3 adjustments" by P.Y. Groisman et al.: Journal of Geophysical Research-D: Atmospheres, v. 103, no. D6, p. 6,221-6,227.

Yang, Daqing, Goodison, B.E., Metcalfe, J.R., Louie, P.Y.T., Elomaa, Esko, Hanson, C.L., Golubev, V.S., Gunther, Thilo, Milkovic, Janja, and Lapan, Milan, 2001, Compatibility evaluation of national precipitation gage measurements: Journal of Geophysical Research-D: Atmospheres, v. 106, no. D2, February 1, p. 1481-1491.

Yang, Daqing, Goodison, B.E., Metcalfe, J.R., Louie, P.Y.T., Leavesley, George, Emerson, D.G., Hanson, C.L., Golubev, V.S., Elomaa, Esko, Gunther, Thilo, Pangburn, Timothy, Kang, Ersi, and Milkovic, Janja, 1999, Quantification of precipitation measurement discontinuity induced by wind shields on national gauges: Water Resources Research, v. 35, no. 2, February, p. 491-508.

Yang, Daqing, Metcalfe, J.R., Goodison, B.E., and Mekis, E., 1993, "True Snowfall:" An evaluation of the double fence intercomparison reference gauge, in 50th Eastern Snow Conference and 61st Western Snow Conference, Quebec City, Canada, June 8-18, 1993, Proceedings: Eastern Snow Conference and Western Snow Conference, p. 105-111.

World Meteorological Organization/Commission for Instruments and Methods of Observation, 1993, International Organizing Committee for the WMO Solid Precipitation Measurement Intercomparison session, 6th, Toronto, Canada, Final Report: Geneva, Switzerland, World Meteorological Organization, $15 \mathrm{p}$.

Publishing support provided by:

Helena Publishing Service Center

For more information concerning this publication, contact: Director, USGS North Dakota Water Science Center

$821 \mathrm{E}$. Interstate Ave.

Bismarck, ND 58503

(701) 250-7400

Or visit the North Dakota Water Science Center Web site at: http://nd.water.usgs.gov 



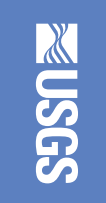

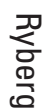

을

产

Printed on recycled paper 